Working Paper/Document de travail 2015-39

\title{
Option Valuation with Observable Volatility and Jump Dynamics
}

by Peter Christoffersen, Bruno Feunou and Yoontae Jeon 
Bank of Canada Working Paper 2015-39

November 2015

\title{
Option Valuation with Observable Volatility and Jump Dynamics
}

by

\section{Peter Christoffersen, ${ }^{1}$ Bruno Feunou ${ }^{2}$ and Yoontae Jeon ${ }^{3}$}

\author{
${ }^{1}$ Rotman School of Management \\ University of Toronto \\ CBS and CREATES \\ Corresponding author: peter.christoffersen@rotman.utoronto.ca \\ 2Financial Markets Department \\ Bank of Canada \\ Ottawa, Ontario, Canada K1A 0G9 \\ feun@bankofcanada.ca \\ ${ }^{3}$ Rotman School of Management \\ University of Toronto
}




\section{Acknowledgements}

We are grateful to the Bank of Canada, the Global Risk Institute, IFSID, SSHRC and TMX for financial support. Thanks also to Glen Keenleyside for editorial support. 


\begin{abstract}
Under very general conditions, the total quadratic variation of a jump-diffusion process can be decomposed into diffusive volatility and squared jump variation. We use this result to develop a new option valuation model in which the underlying asset price exhibits volatility and jump intensity dynamics. The volatility and jump intensity dynamics in the model are directly driven by model-free empirical measures of diffusive volatility and jump variation. Because the empirical measures are observed in discrete intervals, our option valuation model is cast in discrete time, allowing for straightforward filtering and estimation of the model. Our model belongs to the affine class, enabling us to derive the conditional characteristic function so that option values can be computed rapidly without simulation. When estimated on S\&P500 index options and returns, the new model performs well compared with standard benchmarks.
\end{abstract}

JEL classification: $G, G 1, G 12$

Bank classification: Asset pricing

\title{
Résumé
}

Dans des conditions très générales, la variation quadratique totale d'un processus de diffusion à sauts peut se diviser entre la volatilité de la diffusion et la variation des sauts. Ce résultat permet de construire un nouveau modèle d'évaluation des options où la volatilité et l'intensité des sauts du prix de l'actif sous-jacent varient. Dans ce modèle, les dynamiques de la volatilité et de l'intensité des sauts sont tributaires de mesures non paramétriques de la volatilité de la diffusion et de la variation des sauts. Ces mesures étant observées dans des intervalles discrets, le modèle d'évaluation est formulé en temps discret. Cette approche permet une filtration et une estimation simples du modèle. Le modèle appartient à la classe des modèles affines : cette propriété permet de déduire une fonction caractéristique conditionnelle et, par conséquent, de calculer rapidement les valeurs des options en évitant le recours à des méthodes de simulation. Estimé à partir de rendements et des prix d'options de l'indice S\&P 500, le nouveau modèle offre de bons résultats par rapport aux modèles de référence habituels.

Classification JEL : G, G1, G12

Classification de la Banque : Évaluation des actifs 


\section{Non-Technical Summary}

One of the most important recent developments in the financial econometrics literature is the use of intraday observations to precisely evaluate the variability of the price of any financial asset on a given day. That estimate is commonly known as the "realized variance." Using the realized variance, we can evaluate, for instance, the effect of a policy announcement or macroeconomic news on the uncertainty of a given asset's price.

Large movements in asset prices tend to be less frequent and short lived, while small movements are more frequent and cluster over time. Different types of news and announcements affect these two movements differently. Hence, it is of interest to academics and policy-makers to measure how much variability is attributable to normal or continuous movements in prices versus abnormal or discontinuous movements. Looking at intraday trading activities, researchers have provided us with a decomposition of realized variance as the sum of continuous and discontinuous variation.

This paper evaluates the economic significance of that decomposition by evaluating the mispricing of S\&P500 derivatives under two scenarios: ignoring or using the decomposition of the realized variance. We find that the split is very informative for options pricing. 


\section{Introduction}

State-of-the-art derivative valuation models assume that price changes in the underlying asset are driven by a diffusive component as well as a jump component. ${ }^{1}$ The volatility of the diffusive component is typically assumed to be stochastic and the jump intensity is often assumed to be constant. The econometric literature has developed powerful model-free methods for detecting statistically significant jumps and for separating the daily total diffusive volatility from the total quadratic variation via the use of high-frequency observations. ${ }^{2}$ Our contribution is to combine these insights and develop a new derivative valuation model that directly uses the observable realized diffusive volatility and realized jump variation to model dynamics in the diffusive volatility and in the jump intensity.

We cast our model within the broad class of affine discrete-time models, which implies that volatility and jump intensity filtering is straightforward and that derivative valuation can be done without relying on simulation-based methods. We develop a stochastic discount factor for the model that enables us to compute European option values using Fourier inversion of the conditional characteristic function.

The development of rigorous statistical foundations for the use of intraday returns to construct daily realized volatility measures is arguably one of the most successful branches of financial econometrics. For early references, see Andersen and Bollerslev (1998), BarndorffNielsen and Shephard (2002), Andersen, Bollerslev, Diebold, and Labys (2003), and Zhang, Mykland, and Aït-Sahalia (2005). For an early application of realized volatility in finance, see for example Bakshi, Cao and Chen (1997).

The finance literature has recently developed models that use daily total quadratic variation from intraday data to specify and estimate daily models of option valuation which outperform models estimated only on daily returns. See for example Stentoft (2008), Corsi, Fusari and La Vecchia (2013), and Christoffersen, Feunou, Jacobs and Meddahi (2014). However, we are the first to develop an option valuation model with separate dynamics for observable realized diffusive volatility and realized jump variation.

The econometric literature has shown that decomposing total quadratic variation into its diffusive and jump variation parts leads to improved forecasts of future volatility. See for example Andersen, Bollerslev, and Diebold (2007), and Busch, Christensen, and Nielsen (2011). ${ }^{3}$ Our goal is to assess whether the improvements found in the volatility forecasting

\footnotetext{
${ }^{1}$ See for example Bates (2000, 2012), Eraker (2004), Huang and Wu (2004), and Santa-Clara and Yan (2010).

${ }^{2}$ See Barndorff-Nielsen and Shephard (2004, 2006), Huang and Tauchen (2005), and the recent survey in Aït-Sahalia and Jacod (2012).

${ }^{3}$ Dynamic models for daily returns and volatility using high-frequency information have been developed in Forsberg and Bollerslev (2002), Engle and Gallo (2006), Bollerslev, Kretschmer, Pigorsch, and Tauchen
} 
literature carry over to option valuation. We find that they do.

Our paper is part of a larger research agenda applying realized volatility measures in financial decision making. The development of tools for computing highly informative daily realized volatility from noisy intraday data is arguably one of the great success stories of financial econometrics. The application of realized volatility in option valuation is particularly intriguing. For many assets, including equities, the underlying contract is traded very actively at a high frequency, whereas the option is typically traded much less often (see, for example, Christoffersen, Goyenko, Jacobs, and Karoui, 2014). This setting is ideal for the application of realized volatility, which presumes that the frequency of interest (say daily) is lower than the frequency of observation (say each minute). Realized volatility and jumps, which are generally unobserved at a 1-minute frequency, can be reliably estimated at a daily frequency. This insight enables the implementation of a new class of option valuation models, which we develop in this paper.

When estimating the new model on returns, realized diffusive volatility, and realized jump variation, we find that it outperforms standard benchmark models in the literature, including the Heston and Nandi (2000) affine GARCH model, which is a special case of our model. The general model also outperforms a special case that models only the total quadratic variation dynamic, as well as a special case that assumes the entire quadratic variation is attributable to the jump component.

When estimating the new model on S\&P500 index options as well as returns and realized variation measures and evaluating the option fit, the model again performs well. The option implied volatility root mean squared error of the new model is $17 \%$ below that of the affine GARCH model. The improvement in option fit arises in virtually all the moneyness, maturity and market volatility categories that we consider.

One key advantage of our approach is that we avoid the filtering issues that arise in related discrete time jump models. ${ }^{4}$ More generally, we argue that using high-frequency information to discern between daily jumps and diffusive volatility is likely to lead to a much more accurate identification of the two components than relying only on daily returns, or only on daily returns and options.

The remainder of the paper proceeds as follows: in Section 2 we briefly review the general theory for separating diffusive volatility from jumps and we show the two time series for the S\&P500 index, which is the underlying asset in our empirical study. In Section 3 we develop the physical return process. Section 4 estimates the physical process on returns, realized

(2009), Shephard and Sheppard (2010), and Hansen, Huang, and Shek (2012).

${ }^{4}$ See, for example, Maheu and McCurdy (2004), Christoffersen, Jacobs, and Ornthanalai (2012), and Ornthanalai (2014). 
bipower and jump variation measures. In Section 5 , we derive an option valuation formula for the model, estimate the model on options and analyze its fit. Finally, Section 6 concludes. The proofs of our propositions are relegated to the appendix.

\section{Daily Returns and Realized Variation Measures}

In this section we first briefly review the key theoretical results that allow us to separate daily diffusive volatility and jump variation using intraday data. We then construct empirical measures of realized diffusive volatility and realized jumps, plot the daily realized variation series along with daily returns, and investigate their dynamics.

\subsection{Separating Volatility and Jumps: Theory}

Barndorff-Nielsen and Shephard (2004) assume that the stock price follows a jump-diffusion process of the form

$$
d \log \left(S_{t}\right)=\sqrt{V}_{t} d W_{t}+J_{t} d q_{t},
$$

where $q_{t}$ is a Poisson process with intensity $\lambda_{J}$, and $J_{t}$ is the normally distributed log jump size with mean $\mu_{J}$ and standard deviation $\sigma_{J}$. Under this very general assumption about the instantaneous return process, Barndorff-Nielsen and Shephard (2004) show the following limit result as the sampling frequency goes to infinity:

$$
\begin{aligned}
R V_{t} & \rightarrow \int_{t-1}^{t} V_{s} d s+\int_{t-1}^{t} J_{s}^{2} d q_{s} \\
R B V_{t} & \rightarrow \int_{t-1}^{t} V_{s} d s,
\end{aligned}
$$

where $R V_{t}$ denotes realized variance measuring total quadratic variation, and $R B V_{t}$ denotes bipower variation measuring diffusive volatility. These quantities will be defined in detail below. We can define realized jump variation $\left(R J V_{t}\right)$ using

$$
R J V_{t} \equiv R V_{t}-R B V_{t} \rightarrow \int_{t-1}^{t} J_{s}^{2} d q_{s},
$$

which provides the decomposition of total quadratic variation that we need.

The next step in our analysis is to construct empirical measures of $R V_{t}, R B V_{t}$ and $R J V_{t}$. 


\subsection{Separating Volatility and Jumps: Empirics}

Our empirical investigation begins by obtaining intraday S\&P500 cash index data from TickData.com. Using the last observation each minute, we construct a grid of 1-minute equity index values each day, from which we compute five series of overlapping 5-minute logreturns. Each day we can compute five realized variance measures from the sum of squared 5 -minute returns. The five overlapping realized variance series are then averaged to obtain a single market microstructure robust measure of total quadratic variation, as follows:

$$
R V_{t+1}^{\prime}=\frac{1}{5} \sum_{i=0}^{4} R V_{t+1}^{5, i}=\frac{1}{5} \sum_{i=0}^{4} \sum_{j=1}^{m / 5} R_{t+(i+5 j) / m}^{2}
$$

where $R_{t+(i+5 j) / m}$ denotes the $j^{t h}$ period 5-minute intraday return, and $m$ denotes the number of 1-minute returns available on day $t+1$. Following Hansen and Lunde (2005) the $R V_{t+1}$ computed above is finally rescaled so that the average value of $R V_{t+1}$ is equal to the sample variance of daily log returns:

$$
R V_{t+1}=\frac{\sum_{t=1}^{T} R_{t}^{2}}{\sum_{t=1}^{T} R V_{t}^{\prime}} R V_{t+1}^{\prime}
$$

where $R_{t}=\log \left(S_{t}\right)-\log \left(S_{t-1}\right)$ is the daily log return computed from closing prices.

Diffusive volatility is computed using realized bipower variation defined from

$$
R B V_{t+1}^{\prime}=\frac{1}{5} \sum_{i=0}^{4} R B V_{t+1}^{5, i}=\frac{1}{5} \sum_{i=0}^{4} \frac{\pi}{2} \sum_{j=1}^{m / 5-1}\left|R_{t+(i+5 j) / m}\right|\left|R_{t+(i+5(j+1)) / m}\right|
$$

Then, in order to ensure that the empirical version of the theoretical relationship in equation (2) holds, namely,

$$
R V_{t+1}=R B V_{t+1}+R J V_{t+1}
$$

and also in order to ensure that $R J V_{t+1} \geq 0$, we use the following definitions:

$$
\begin{aligned}
R B V_{t+1} & =\min \left(R V_{t+1}, R B V_{t+1}^{\prime}\right) \\
R J V_{t+1} & =R V_{t+1}-R B V_{t+1} .
\end{aligned}
$$

Figure 1 plots the $R_{t}$ (top left), $R V_{t}$ (top right), $R B V_{t}$ (bottom left) and $R J V_{t}$ (bottom right) series from 2 January 1990 through 31 December 2013. Note from Figure 1 that the $R V_{t}, R B V_{t}$ and $R J V_{t}$ series share broadly similar patterns, including the fact that their largest values occur during the 2008 financial crisis. This commonality suggests that when 
$R V_{t}$ is high then both $R B V_{t}$ and $R J V_{t}$ are high, and vice versa. Note also that $R B V_{t}$ is an order of magnitude larger than $R J V_{t}$.

Figure 2 plots the sample autocorrelation functions for the four series. Note that, as expected, the autocorrelations of returns (top left) are close to zero across lag orders. Also as expected, the autocorrelations of realized variance (top right) and bipower variation (bottom left) are both very high and statistically significant throughout the 60 trading days considered. More interestingly, the realized jump variation measure in the bottom right panel shows strong evidence of persistence as well. To be sure, the autocorelations for realized jump variation are lower at short lags than for realized variance and bipower variation, but they are very persistent. It is thus clear that the realized jump measure requires a dynamic specification of its own and likely one that is different from the dynamic specification of bipower variation. Building a dynamic return model with such features is our next task.

\section{A New Dynamic Model for Asset Returns}

The goal of this section is to build a model for end-of-day $t$ option valuation that incorporates the information in the $R_{t}, R B V_{t}$ and $R J V_{t}$ series computed at the end of the day. We want to build a model in which state variables are explicitly filtered using our observables and in which option valuation can be done without Monte Carlo simulation.

\subsection{The Asset Return Process}

Consider first the following generic specification of daily log returns:

$$
R_{t+1}=r+\left(\lambda_{z}-\frac{1}{2}\right) h_{z, t}+\left(\lambda_{y}-\xi\right) h_{y, t}+z_{t+1}+y_{t+1}
$$

where $r$ denotes the risk-free rate, and the first innovation, $z_{t+1}$, denotes a heteroskedastic Gaussian innovation:

$$
z_{t+1}=\sqrt{h_{z, t}} \varepsilon_{1, t+1} \text {, with } \varepsilon_{1, t+1} \stackrel{i i d}{\sim} N(0,1)
$$

The second innovation, $y_{t+1}$, denotes a compound jump process,

$$
y_{t+1}=\sum_{j=0}^{n_{t+1}} x_{t+1}^{j}, \text { with } x_{t+1}^{j} \stackrel{i i d}{\sim} N\left(\theta, \delta^{2}\right)
$$


where the number of Gaussian jumps per day is Poisson distributed:

$$
n_{t+1} \sim P s\left(h_{y, t}\right) .
$$

Note that this general framework allows for dynamic volatility via $h_{z, t}$ and dynamic jump intensity via $h_{y, t}$. These dynamics still need to be specified and, crucially for us, they need to be linked with the daily realized bipower and jump variation measures.

Finally, note that in our timing convention, $h_{z, t}$ denotes the expected "diffusive" variance for day $t+1$ constructed at the end of day $t$. Similarly, $h_{y, t}$ denotes the expected number of jumps on day $t+1$ constructed at the end of day $t$.

The parameters we estimate on daily data in our discrete time model are reported in daily units below. When estimating continuous time models of the type in (1), the literature often reports annualized parameters. In that case, we have the following mapping:

$$
\theta \approx \frac{1}{252} \mu_{J}, \quad \delta \approx \frac{1}{\sqrt{252}} \sigma_{J}, \quad h_{y, t} \approx \frac{1}{252} \int_{t-1}^{t} \lambda_{J, s} d s .
$$

\subsection{Incorporating Realized Bipower and Jump Variation}

Each day the realized bipower variation provides new information about diffusive volatility, $h_{z, t}$. However, $R B V_{t+1}$ is measured with error and we therefore specify the following measurement equation:

$$
R B V_{t+1}=h_{z, t}+\sigma\left[\left(\varepsilon_{2, t+1}-\gamma \sqrt{h_{z, t}}\right)^{2}-\left(1+\gamma^{2} h_{z, t}\right)\right]
$$

where we have introduced a measurement error variable,

$$
\varepsilon_{2, t+1} \stackrel{i i d}{\sim} N(0,1)
$$

which has a correlation $\rho$ with the diffusive return shock, $\varepsilon_{1, t+1}$, defined in equation (4). The innovation term inside the brackets in equation (7) is constructed to have zero mean, ensuring that

$$
E_{t}\left[R B V_{t+1}\right]=h_{z, t} .
$$

Note also that equation (7) allows for a non-linear impact of $\varepsilon_{2, t+1}$ on $R B V_{t+1}$ via $\gamma$.

Our daily realized jump variation measure constructed from intraday data is naturally 
linked with the sum of squared daily jump variation in the model as follows:

$$
R J V_{t+1}=\sum_{j=0}^{n_{t+1}}\left(x_{t+1}^{j}\right)^{2} .
$$

This relationship implies that

$$
E_{t}\left[R J V_{t+1}\right]=\left(\theta^{2}+\delta^{2}\right) h_{y, t}
$$

where we have used the second moment of the Poisson distribution. Notice that while we do allow for measurement error in $R B V_{t}$, we assume for simplicity that $R J V_{t}$ is free of error. We plan to relax this assumption in future work.

\subsection{Volatility and Jump Dynamics}

We are ready to specify the dynamics of the expected volatility and jump intensity. In the empirics below, we will focus on a special case of our modelling framework in which we simply pose that

$$
\begin{aligned}
h_{z, t+1} & =\omega_{z}+b_{z} h_{z, t}+a_{z} R B V_{t+1}, \text { and } \\
h_{y, t+1} & =\omega_{y}+b_{y} h_{y, t}+a_{y} R J V_{t+1} .
\end{aligned}
$$

Note that in this specification, $h_{z, t+1}$ and $h_{y, t+1}$ are both univariate $A R(1)$ processes, which we can write as

$$
\begin{aligned}
& h_{z, t+1}=\omega_{z}-a_{z} \sigma+\left(b_{z}+a_{z}-a_{z} \sigma \gamma^{2}\right) h_{z, t}+a_{z} \sigma\left(\varepsilon_{2, t+1}-\gamma \sqrt{h_{z, t}}\right)^{2} \\
& h_{y, t+1}=\omega_{y}+b_{y} h_{y, t}+a_{y} \sum_{j=0}^{n_{t+1}}\left(x_{t+1}^{j}\right)^{2} .
\end{aligned}
$$

The dynamics in (8-9) imply that $R B V_{t+1}$ and $R J V_{t+1}$ are both univariate $A R M A(1,1)$ processes. We will refer to this as the BiPower and Realized Jump Model (BPRJM). 


\subsection{The General Case}

Our dynamic modelling framework is of course more general than the particular BPRJM model. To see this, define the bivariate processes:

$$
\begin{aligned}
h_{t} & \equiv\left(h_{z, t}, h_{y, t}\right)^{\prime}, \text { and } \\
R V M_{t+1} & \equiv\left(R B V_{t+1}, R J V_{t+1}\right)^{\prime} .
\end{aligned}
$$

The general dynamic vector process is then of the form

$$
h_{t+1}=\omega+b h_{t}+a R V M_{t+1}
$$

where the parameter vector and matrices are

$$
\omega=\left(\omega_{z}, \omega_{y}\right)^{\prime}, b=\left(\begin{array}{cc}
b_{z} & b_{z, y} \\
b_{y, z} & b_{y}
\end{array}\right), a=\left(\begin{array}{cc}
a_{z} & a_{z, y} \\
a_{y, z} & a_{y}
\end{array}\right) .
$$

Note that, by construction, $h_{t+1}$ is a vector autoregressive process of order one, $V A R(1)$, and $R V M_{t+1}$ is a vector autoregressive moving average model, $V A R M A(1,1)$. In particular, note that the expected value of the vector $h_{t+1}$ is

$$
\begin{aligned}
E_{t}\left[h_{t+1}\right] & =\omega+b h_{t}+a\left(\begin{array}{c}
h_{z, t} \\
\left(\theta^{2}+\delta^{2}\right) h_{y, t}
\end{array}\right) \\
& \equiv \omega+\left[\begin{array}{ll}
b_{z}+a_{z} & b_{z, y}+\left(\theta^{2}+\delta^{2}\right) a_{z, y} \\
b_{y, z}+a_{y, z} & b_{y}+\left(\theta^{2}+\delta^{2}\right) a_{y}
\end{array}\right] h_{t} .
\end{aligned}
$$

Below we will focus on the BPRJM version of the model in which $a_{z, y}=a_{y, z}=b_{z, y}=$ $b_{y, z}=0$.

\subsection{Expected Returns and Risk Premiums}

It is clear from equation (3) that the one-day-ahead conditionally expected log returns in the model are simply

$$
E_{t}\left[R_{t+1}\right]=r+\left(\lambda_{z}-\frac{1}{2}\right) h_{z, t}+\left(\lambda_{y}-\xi+\theta\right) h_{y, t}
$$

The jump compensator parameter, $\xi$, in our model is itself a particular function of other parameters:

$$
\xi=e^{\theta+\frac{1}{2} \delta^{2}}-1
$$


This functional form ensures that the conditionally expected total return is

$$
E_{t}\left[\exp \left(R_{t+1}\right)\right]=\exp \left(r+\lambda_{z} h_{z, t}+\lambda_{y} h_{y, t}\right)
$$

which in turn ensures that $\lambda_{z}$ and $\lambda_{y}$ can be viewed as compensation for diffusive volatility and jump exposure, respectively. Substituting equation (3) into (11), taking expectations, and solving for $\xi$ yields equation (10). The $\xi$ parameter will therefore not be estimated below, but will instead simply be set to its value implied by equation (10).

\subsection{Conditional Second Moments}

From the model above, it is relatively straightforward to derive the following one-day-ahead conditional second moments:

$$
\begin{aligned}
\operatorname{Var}_{t}\left[R_{t+1}\right] & =h_{z, t}+\left(\theta^{2}+\delta^{2}\right) h_{y, t} \\
\operatorname{Var}_{t}\left[R B V_{t+1}\right] & =2 \sigma^{2}\left(1+2 \gamma^{2} h_{z, t}\right) \\
\operatorname{Var}_{t}\left[R J V_{t+1}\right] & =\left(\theta^{4}+3 \delta^{4}+6 \theta^{2} \delta^{2}\right) h_{y, t} \\
\operatorname{Cov}_{t}\left(R_{t+1}, R B V_{t+1}\right) & =-2 \rho \gamma \sigma h_{z, t} \\
\operatorname{Cov}_{t}\left(R_{t+1}, R J V_{t+1}\right) & =\theta\left(\theta^{2}+3 \delta^{2}\right) h_{y, t} \\
\operatorname{Cov}_{t}\left(R B V_{t+1}, R J V_{t+1}\right) & =0 .
\end{aligned}
$$

Note that the model allows for two types of "leverage" effects: one via the return covariance with bipower variation, and another via the return covariance with jumps.

\section{Physical Parameter Estimates}

Above we have laid out the general framework for incorporating bipower variation and realized jump variation when modelling return dynamics. In this section we develop a likelihoodbased estimation method that enables us to estimate the physical parameters using daily observations on returns, as well as the realized variation measures from Figure 1. We also develop two special cases of the general model and briefly describe the Heston and Nandi (2000) benchmark GARCH model. 


\subsection{Deriving the Likelihood Function}

When deriving the conditional quasi-likelihood function, note first that the contribution to the total conditional likelihood by day $t+1$ can be obtained by summing over the number of jumps occurring on that day. We can write

$$
\begin{aligned}
f_{t}\left(R_{t+1}, R B V_{t+1}, R J V_{t+1}\right) & =\sum_{j=0}^{\infty} f_{t}\left(R_{t+1}, R B V_{t+1}, R J V_{t+1}, n_{t+1}=j\right) \\
& =\sum_{j=0}^{\infty} f_{t}\left(R_{t+1}, R B V_{t+1}, R J V_{t+1} \mid n_{t+1}=j\right) P_{t}\left[n_{t+1}=j\right]
\end{aligned}
$$

with the number of jumps drawn from the Poisson distribution,

$$
P_{t}\left[n_{t+1}=j\right]=\frac{e^{-h_{y, t}} h_{y, t}^{j}}{! j}
$$

Separating out the days with exactly zero jumps, we get

$$
f_{t}\left(R_{t+1}, R B V_{t+1}, R J V_{t+1} \mid n_{t+1}=j\right)=\left\{\begin{array}{ll}
f_{t}\left(R_{t+1}, R B V_{t+1}\right), & \text { if } j=0 \\
f_{t}(j), & \text { if } j>0
\end{array} .\right.
$$

In order to save on notation, define the variable vectors

$$
X_{t+1} \equiv\left(R_{t+1}, R B V_{t+1}, R J V_{t+1}\right)^{\prime} \quad X_{t+1}^{(1,2)} \equiv\left(R_{t+1}, R B V_{t+1}\right)^{\prime}
$$

and the corresponding conditional first and second moments:

$$
\begin{array}{ll}
\mu_{t}\left(n_{t+1}\right) \equiv E_{t}\left[X_{t+1} \mid n_{t+1}\right] & \mu_{t}^{(1,2)} \equiv E_{t}\left[X_{t+1}^{(1,2)} \mid n_{t+1}\right] \\
\Omega_{t}\left(n_{t+1}\right) \equiv \operatorname{Var}_{t}\left[X_{t+1} \mid n_{t+1}\right] & \Omega_{t}^{(1,2)}\left(n_{t+1}\right) \equiv \operatorname{Var}_{t}\left[X_{t+1}^{(1,2)} \mid n_{t+1}\right]
\end{array}
$$

Then we can write the marginal likelihood for returns and bipower variation when $n_{t+1}=$ 0 as

$$
\begin{aligned}
f_{t}\left(R_{t+1}, R B V_{t+1}\right)= & (2 \pi)^{-1}\left|\Omega_{t}^{(1,2)}(0)\right|^{-1 / 2} \\
& \cdot \exp \left(-\frac{1}{2}\left(X_{t+1}^{(1,2)}-\mu_{t}^{(1,2)}(0)\right)^{\prime} \Omega_{t}^{(1,2)}(0)^{-1}\left(X_{t+1}^{(1,2)}-\mu_{t}^{(1,2)}(0)\right)\right),
\end{aligned}
$$


and when $n_{t+1}>0$ we have

$$
f_{t}(j)=(2 \pi)^{-3 / 2}\left|\Omega_{t}(j)\right|^{-1 / 2} \exp \left(-\frac{1}{2}\left(X_{t+1}-\mu_{t}(j)\right)^{\prime} \Omega_{t}(j)^{-1}\left(X_{t+1}-\mu_{t}(j)\right)\right) .
$$

The log-likelihood is then defined by

$$
\ln L^{P}=\sum_{t=1}^{T-1} \ln \left(f_{t}\left(R_{t+1}, R B V_{t+1}, R J V_{t+1}\right)\right) .
$$

We maximize the likelihood function in (14) using the fminunc routine in Matlab with the following settings:

optimset('display','iter','MaxIter',1500,'TolFun',1e-5,'MaxFunEvals',1e+06,'TolX',1e-20).

\subsection{Conditional Moments}

The likelihood function above requires that we derive the first two moments conditional on time and on the number of jumps, $n_{t+1}$. For the conditional first moments we have

$$
\begin{aligned}
E_{t}\left[R_{t+1} \mid n_{t+1}\right] & =r+\left(\lambda_{z}-\frac{1}{2}\right) h_{z, t}+\left(\lambda_{y}-\xi\right) h_{y, t}+\theta n_{t+1} \\
E_{t}\left[R B V_{t+1} \mid n_{t+1}\right] & =h_{z, t} \\
E_{t}\left[R J V_{t+1} \mid n_{t+1}\right] & =\left(\theta^{2}+\delta^{2}\right) n_{t+1} .
\end{aligned}
$$

For the conditional second moments we have

$$
\begin{aligned}
\operatorname{Var}_{t}\left[R_{t+1} \mid n_{t+1}\right] & =h_{z, t}+\delta^{2} n_{t+1} \\
\operatorname{Var}_{t}\left[R B V_{t+1} \mid n_{t+1}\right] & =2 \sigma^{2}\left(1+2 \gamma^{2} h_{z, t}\right) \\
\operatorname{Var}_{t}\left[R J V_{t+1} \mid n_{t+1}\right] & =2 \delta^{2}\left(\delta^{2}+2 \theta^{2}\right) n_{t+1} \\
\operatorname{Cov}_{t}\left[R_{t+1}, R B V_{t+1} \mid n_{t+1}\right] & =-2 \rho \gamma \sigma h_{z, t} \\
\operatorname{Cov}_{t}\left[R_{t+1}, R J V_{t+1} \mid n_{t+1}\right] & =2 \theta \delta^{2} n_{t+1} \\
\operatorname{Cov}_{t}\left[R B V_{t+1}, R J V_{t+1} \mid n_{t+1}\right] & =0 .
\end{aligned}
$$

From these moments we can easily construct the $\mu_{t}$ vectors and $\Omega_{t}$ matrices in equation (13) needed for the likelihood function in equation (14).

Before turning to estimation of the new model, we define three special cases of interest which we also estimate below. 


\subsection{The Heston-Nandi GARCH Model as a Special Case}

First, by setting $h_{y, t}=0$, and $\rho=1$, we obtain one of the standard $\operatorname{GARCH}(1,1)$ models in the literature. Specifically, note that $\rho=1$ implies that $\varepsilon_{1, t+1}=\varepsilon_{2, t+1}$, and the realized variance therefore becomes irrelevant. We then get

$$
\begin{aligned}
h_{z, t+1} & =\omega_{z}-a_{z} \sigma+\left(b_{z}+a_{z}-a_{z} \sigma \gamma^{2}\right) h_{z, t}+a_{z} \sigma\left(\varepsilon_{2, t+1}-\gamma \sqrt{h_{z, t}}\right)^{2} \\
& \equiv \omega+\beta h_{z, t}+\alpha\left(\varepsilon_{1, t+1}-\gamma \sqrt{h_{z, t}}\right)^{2},
\end{aligned}
$$

which is exactly the Heston and Nandi (2000) affine GARCH(1,1) model.

\subsection{The realized volatility model (RVM) as a Special Case}

Second, we can shut down the separate jump variation by setting $h_{y, t}=0$ in the new model. We then get

$$
\begin{aligned}
R_{t+1} & \equiv \log \left(\frac{S_{t+1}}{S_{t}}\right)=r+\left(\lambda_{z}-\frac{1}{2}\right) h_{z, t}+z_{t+1}, \text { with } z_{t+1}=\sqrt{h_{z, t}} \varepsilon_{1, t+1} \\
R V_{t+1} & =R B V_{t+1}+R J V_{t+1}=h_{z, t}+\sigma\left[\left(\varepsilon_{2, t+1}-\gamma \sqrt{h_{z, t}}\right)^{2}-\left(1+\gamma^{2} h_{z, t}\right)\right] .
\end{aligned}
$$

This is exactly the autoregressive RV model in Christoffersen, Feunou, Jacobs, and Meddahi (2014). We will refer to this as the RVM model below.

\subsection{The realized jump model (RJM) as a Special Case}

Third, we can shut down the bipower variation channel by setting $h_{z, t}=0$. We then get

$$
\begin{aligned}
R_{t+1} & =r-\theta-\frac{\delta^{2}}{2}+\left(\lambda_{y}-\xi\right) h_{y, t}+y_{t+1} \\
y_{t+1} & =\sum_{j=1}^{n_{t+1}} x_{t+1}^{j}, \text { where } x_{t+1}^{j} \stackrel{i i d}{\sim} N\left(\theta, \delta^{2}\right) \\
P\left[n_{t+1}\right. & \left.=j \mid I_{t}\right]=\frac{e^{-h_{y, t}} h_{y, t}^{j-1}}{!(j-1)},
\end{aligned}
$$

and furthermore we set

$$
\begin{aligned}
R V_{t+1} & =\sum_{j=1}^{n_{t+1}}\left(x_{t+1}^{j}\right)^{2}-\theta^{2} \\
h_{y, t+1} & =\omega_{y}+b_{y} h_{y, t}+a_{y} R V_{t+1} .
\end{aligned}
$$


Note that in this case the entire quadratic variation is assumed to be driven by jumps, so that each day has at least one jump. We will refer to this as the RJM model below.

\subsection{Parameter Estimates and Model Properties}

Table 1 contains the maximum likelihood estimation results for the physical return processes developed above. One year prior to our estimation sample, we set the conditional variance equal to the unconditional variance and then burn-in the model on the pre-sample year to get an appropriate conditional variance on the first day of the sample. Note that the $\omega$ parameters do not have standard errors, since they are computed by variance targeting, thus exactly matching the observed sample variance of returns. The parameter estimates are generally significant except for $\lambda s$, which are always difficult to pin down in relatively short return-based samples.

Note that volatility persistence is very high in the RVM and BPRJM models, and considerably lower in the GARCH and RJM models. Unconditional volatility and volatility persistence is defined in the GARCH model as

$$
E\left[h_{t}\right]=\frac{\omega+\alpha}{1-\left(\beta+\alpha \gamma^{2}\right)} \equiv \frac{\omega+\alpha}{1-\text { Persist }}
$$

in the RVM model as

$$
E\left[h_{z, t}\right]=\frac{\omega_{z}}{1-\left(b_{z}+a_{z}\right)} \equiv \frac{\omega_{z}}{1-\text { Persist }}
$$

in the RJM model as

$$
E\left[h_{y, t}\right]=\frac{\omega_{y}}{1-\left(b_{y}+\left(\theta^{2}+\delta^{2}\right) a_{y}\right)} \equiv \frac{\omega_{y}}{1-\text { Persist }}
$$

and in the BPRJM model as

$$
E\left[h_{t}\right]=\frac{\omega_{z}}{1-\left(b_{z}+a_{z}\right)}+\frac{\left(\theta^{2}+\delta^{2}\right) \omega_{y}}{1-\left(b_{y}+\left(\theta^{2}+\delta^{2}\right) a_{y}\right)} .
$$

Persistence for the two variance components in the BPRJM model is thus equal to the RVM and RJM cases.

When comparing model fit, we are faced with the challenge that the GARCH model is only fit to returns, the RVM and RJM models are fit to returns and RV, and the general BPRJM model is fit to returns, $R B V$ and $R J V$. Table 1 shows that the likelihood value for the general model is 129,226 , but this is not readily comparable to the other models which are fit to different quantities. We therefore re-estimate the BPRJM model maximizing only the 
joint likelihood of returns and RV. ${ }^{5}$ The second row of log-likelihoods contains the results. From this perspective, the BPRJM model by far performs the best, with a likelihood of 69,656 compared with 68,783 for the RJM model and 68,212 for the RVM model.

When maximizing only the return likelihood, the BPRJM model again performs the best, with a likelihood of 19,522. The improvement over the RVM and RJM models is not dramatic here, but returns are unlikely to be informative about all the parameters of the model, and so this set of results is provided only to enable comparison with the GARCH model. Note that the RVM, RJM and BPRJM models all perform very well compared with the benchmark affine GARCH model.

In Figure 3 we plot the daily conditional volatility, computed as the square root of $h_{t+1}$ for each model. Note that the volatility spikes are much more dramatic in the RVM, RJM and BPRJM models than in the GARCH model. It is interesting and perhaps surprising that the RJM model is able to produce a spot volatility time path which is quite similar to that from the RVM and BPRJM models. This is partly because the RJM model is fit to returns and $R V$, and not to returns and $R J V$.

In Figure 4 we plot the daily conditional volatility of variance computed as the square root of

$$
\operatorname{Var}_{t}\left(h_{t+1}\right)=2 a_{z}^{2} \sigma^{2}\left(1+2 \gamma^{2} h_{z, t}\right)+a_{y}^{2}\left(\theta^{2}+\delta^{2}\right)^{2}\left(\left(2 \delta^{2}\left(\theta^{2}+2 \delta^{2}\right)+\left(\theta^{2}+\delta^{2}\right)^{2}\right) h_{y, t}\right.
$$

for the BPRJM model. The variance of variance expressions for the other models is similar. Note from Figure 4 that the conditional volatility of variance is relatively low and almost constant in the GARCH model, whereas in the other models it tends to be large when volatility is high, thus matching the empirical evidence. Note that the volatility of volatility is slightly lower in the RJM than in the RVM and BPRJM models.

In Figure 5 we plot the conditional correlation of returns and variance, which are computed for the BPRJM model using

$$
\operatorname{Corr}_{t}\left(R_{t+1}, h_{t+1}\right)=\frac{-2 \rho \sigma \gamma a_{z} h_{z, t}+a_{y} \theta\left(\theta^{2}+\delta^{2}\right)\left(\theta^{2}+3 \delta^{2}\right) h_{y, t}}{\sqrt{\operatorname{Var}_{t}\left[R_{t+1}\right] \operatorname{Var}_{t}\left(h_{t+1}\right)}},
$$

where the terms in the denominator can be obtained from equations (12) and (15). The conditional correlation expressions for the other models are similar. Figure 5 shows that the differences across models are quite large from this perspective. The GARCH model implies a correlation of almost negative one. The other models imply correlations of around -0.2 . The RJM and the BPRJM models imply some time-series variation in the correlation, whereas

\footnotetext{
${ }^{5}$ See Appendix A for the details.
} 
the RJM model does not.

The negative correlation between return and volatility is a strong empirical regularity. It is often termed the "leverage" effect, but there is actually not much evidence that it is driven by financial leverage (see, for example, Hasanhodzic and Lo, 2011), and its economic source is as yet largely unknown. In our models, the negative daily correlation generates negative skewness across horizons, which in turn creates higher prices for out-of-the-money puts (and in-the-money calls), which the Black-Scholes model is well-known to underprice. In the Heston-Nandi GARCH model, a large estimate of $\gamma$ generates the very large negative correlation evident in Figure 5, but it is also needed to generate variance of variance dynamics in that model. We suspect that the large value of $\gamma$ in the Heston-Nandi GARCH model is partly driven by the need for a sufficiently high variance of variance (and thus kurtosis) and that it in turn causes the perhaps unrealistically large negative "leverage" correlation seen in Figure 5.

Figure 6 presents evidence on the different models' ability to forecast one-day-ahead realized variance. The ex-post realized variance is on the vertical axis and the model-predicted variance is on the horizontal axis. The corresponding regression fit is $49 \%$ for the GARCH model, $85 \%$ for the RJM model and $87 \%$ for the RVM and BPRJM models. ${ }^{6}$ The slope coefficient on the volatility forecast, which ideally should be 1 , is 2.5 in the GARCH model, 1.3 in the RJM model and 1.1 in the RVM and BPRJM models. The RVM and BPRJM models are thus able to predict realized variance quite well.

The physical properties we have investigated above are likely to have important implications for the models' ability to fit large panels of options. This is the task to which we next turn.

\section{Option Valuation}

In this section we show how the physical model developed above can be used for option valuation. We first derive the moment-generating function of the physical process and show that it is affine. We then define a pricing kernel which implies that the risk-neutral momentgenerating function is of the same form as its physical counterpart. This in turn implies that we can compute option prices using Fourier inversion. The following subsections report empirical results of estimating the model jointly on returns, realized measures and options.

\footnotetext{
${ }^{6}$ The detailed regression results are not reported in the tables, but are available from the authors upon request.
} 


\subsection{The Physical Moment-Generating Function}

Using the vector notation $h_{t} \equiv\left(h_{z, t}, h_{y, t}\right)^{\prime}$ defined above, and further defining the coefficients $v \equiv\left(v_{z}, v_{y}\right)^{\prime}$, Appendix B shows that we can write the physical moment-generating function (MGF) as

$$
\begin{aligned}
E_{t}\left[\exp \left(u R_{t+1}+v^{\prime} h_{t+1}\right)\right] & =\exp \left(\begin{array}{c}
u\left(r+\left(\lambda_{z}-\frac{1}{2}\right) h_{z, t}+\left(\lambda_{y}-\xi\right) h_{y, t}\right)+v^{\prime}\left(\omega+b h_{t}\right) \\
+v_{1}\left(h_{z, t}-\sigma\left(1+\gamma^{2} h_{z, t}\right)\right)-\frac{1}{2} \ln \left(1-2 \sigma v_{1}\right) \\
+\left(v_{1} \sigma \gamma^{2}+\frac{1}{2}\left(1-\rho^{2}\right) u^{2}+\frac{\left(u \rho-2 \sigma v_{1} \gamma\right)^{2}}{2\left(1-2 \sigma v_{1}\right)}\right) h_{z, t}+\left(e^{v_{3}}-1\right) h_{y, t}
\end{array}\right) \\
\equiv & \exp \left(A(u, v)^{\prime} h_{t}+B(u, v)\right)
\end{aligned}
$$

where we have further defined

$$
v^{\prime} a=\left(v_{z}, v_{y}\right)\left(\begin{array}{cc}
a_{z} & a_{z, y} \\
a_{y, z} & a_{y}
\end{array}\right)=\left(v_{z} a_{z}+v_{y} a_{y, z}, v_{z} a_{z, y}+v_{y} a_{y}\right) \equiv\left(v_{1}, v_{2}\right)
$$

and

$$
v_{3}=-\frac{1}{2} \ln \left(1-2 v_{2} \delta^{2}\right)+u \theta+v_{2} \theta^{2}+\frac{\left(u+2 \theta v_{2}\right)^{2} \delta^{2}}{2\left(1-2 v_{2} \delta^{2}\right)}
$$

Note that the physical MGF is of an exponentially affine form, which will greatly facilitate option valuation below.

\subsection{Risk Neutralization}

We follow Christoffersen, Elkamhi, Feunou, and Jacobs (2010) and assume an exponential pricing kernel of the form

$$
\begin{aligned}
\zeta_{t+1} & =\frac{M_{t+1}}{E_{t}\left[M_{t+1}\right]} \equiv \frac{\exp \left(\nu_{1, t} \varepsilon_{1, t+1}+\nu_{2, t} \varepsilon_{2, t+1}+\nu_{3, t} \sum_{j=0}^{n_{t+1}} x_{t+1}^{j}\right)}{E_{t}\left[\exp \left(\nu_{1, t} \varepsilon_{1, t+1}+\nu_{2, t} \varepsilon_{2, t+1}+\nu_{3, t} \sum_{j=0}^{n_{t+1}} x_{t+1}^{j}\right)\right]} \\
& =\exp \left(\begin{array}{c}
\nu_{1, t} \varepsilon_{1, t+1}+\nu_{2, t} \varepsilon_{2, t+1}+\nu_{3, t} \sum_{j=0}^{n_{t+1}} x_{t+1}^{j} \\
\left.-\frac{1}{2} \nu_{1, t}^{2}-\frac{1}{2} \nu_{2, t}^{2}-\rho \nu_{1, t} \nu_{2, t}-\left(e^{\theta \nu_{3, t}+\frac{1}{2} \delta^{2} \nu_{3, t}^{2}}-1\right) h_{y, t}\right)
\end{array}\right.
\end{aligned}
$$

In order to ensure that the model is affine under $Q$, it is necessary and sufficient to impose the following conditions:

$$
\begin{aligned}
& \nu_{2, t}=\left(\gamma-\gamma^{*}\right) \sqrt{h_{z, t}}-\rho \nu_{1, t} \\
& \nu_{3, t}=\nu_{3} .
\end{aligned}
$$


Appendix $\mathrm{C}$ shows that the risk-neutral probability measure for the BPRJM model is then

$$
\begin{aligned}
R_{t+1} & \equiv \log \left(\frac{S_{t+1}}{S_{t}}\right)=r-\frac{1}{2} h_{z, t}-\xi^{*} h_{y, t}^{*}+\sqrt{h_{z, t}} \varepsilon_{1, t+1}^{*}+y_{t+1} \\
y_{t+1} & =\sum_{j=0}^{n_{t+1}} x_{t+1}^{j} ; \quad x_{t+1}^{j} \stackrel{i i d}{\sim}^{Q} N\left(\theta^{*}, \delta^{2}\right) ; n_{t+1} \mid I_{t} \sim^{Q} \text { Poisson }\left(h_{y, t}^{*}\right) \\
R B V_{t+1} & =h_{z, t}+\left(\left(\gamma^{*}\right)^{2}-\gamma^{2}\right) h_{z, t}+\sigma\left[\left(\varepsilon_{2, t+1}^{*}-\gamma^{*} \sqrt{h_{z, t}}\right)^{2}-\left(1+\left(\gamma^{*}\right)^{2} h_{z, t}\right)\right] \\
R J V_{t+1} & =\sum_{j=0}^{n_{t+1}}\left(x_{t+1}^{j}\right)^{2},
\end{aligned}
$$

where $\varepsilon_{1, t+1}^{*}$ and $\varepsilon_{2, t+1}^{*}$ are bivariate Gaussian under $Q$, and where

$$
\begin{aligned}
h_{y, t}^{*} & =e^{\theta \nu_{3}+\frac{1}{2} \delta^{2} \nu_{3}^{2}} h_{y, t} \\
\theta^{*} & =\theta+\delta^{2} \nu_{3}, \quad \xi^{*}=e^{\theta^{*}+\frac{1}{2} \delta^{2}}-1 .
\end{aligned}
$$

Hence we have the risk premiums

$$
\begin{aligned}
E_{t}^{Q}\left[R B V_{t+1}\right]-E_{t}\left[R B V_{t+1}\right] & =\left(\left(\gamma^{*}\right)^{2}-\gamma^{2}\right) h_{z, t} \\
E_{t}^{Q}\left[R J V_{t+1}\right]-E_{t}\left[R J V_{t+1}\right] & =\left(\left(\theta^{*}\right)^{2}+\delta^{2}\right) h_{y, t}^{*}-\left(\theta^{2}+\delta^{2}\right) h_{y, t}
\end{aligned}
$$

where $\gamma^{*}$ and $\nu_{3}$ are additional parameters to be estimated. Below we will use the notation $\chi=\gamma-\gamma^{*}$ and report estimates of $\chi$ instead of $\gamma^{*}$.

By the nature of the model, risk neutralization of the RJM model is slightly different from the other models. Appendix D provides the details.

\subsection{Computing Option Values}

Above we have shown that the risk-neutral distribution is of the same form as physical distribution. The risk-neutral MGF will therefore be of the form shown in Appendix B, but with risk-neutral parameters used instead of their physical counterparts. We can write the 
one-period risk-neutral conditional MGF as

$$
\begin{aligned}
\Psi_{t, t+1}^{Q} & \equiv E_{t}^{Q}\left[\exp \left(u R_{t+1}+v^{\prime} h_{t+1}\right)\right] \\
& =\exp \left(\begin{array}{c}
u\left(r-\frac{1}{2} h_{z, t}-\xi^{*} h_{y, t}^{*}\right)+v^{\prime}\left(\omega+b h_{t}\right) \\
+v_{1}\left(h_{z, t}+\left(\left(\gamma^{*}\right)^{2}-\gamma^{2}\right) h_{z, t}-\sigma\left(1+\left(\gamma^{*}\right)^{2} h_{z, t}\right)\right)-\frac{1}{2} \ln \left(1-2 \sigma v_{1}\right) \\
+\left(v_{1} \sigma\left(\gamma^{*}\right)^{2}+\frac{1}{2}\left(1-\rho^{2}\right) u^{2}+\frac{\left(u \rho-2 \sigma v_{1}^{*} \gamma^{*}\right)^{2}}{2\left(1-2 \sigma v_{1}\right)}\right) h_{z, t}+\left(e^{v_{3}}-1\right) e^{\theta \nu_{3}+\frac{1}{2} \delta^{2} \nu_{3}^{2}} h_{y, t}
\end{array}\right) \\
& \equiv \exp \left(A^{*}(u, v)^{\prime} h_{t}+B^{*}(u, v)\right) .
\end{aligned}
$$

Next, call option values can be computed via standard Fourier inversion techniques,

$$
\begin{aligned}
\text { Call } & =S_{t} P_{1}(t, M)-\exp (-r M) X P_{2}(t, M), \text { where } \\
P_{1}(t, M) & =\frac{1}{2}+\int_{0}^{+\infty} \operatorname{Re}\left(\frac{\Psi_{t, t+M}^{Q}(1+i u) \exp \left(-r M-i u \ln \left(\frac{X}{S_{t}}\right)\right)}{\pi i u}\right) d u \\
P_{2}(t, M) & =\frac{1}{2}+\int_{0}^{+\infty} \operatorname{Re}\left(\frac{\Psi_{t, t+M}^{Q}(i u) \exp \left(-i u \ln \left(\frac{X}{S_{t}}\right)\right)}{\pi i u}\right) d u,
\end{aligned}
$$

where $\Psi_{t, t+M}^{Q}$ denotes the risk-neutral $M$-period MGF (see Appendix B) corresponding to the one-day MGF in equation (19). Put option values can be computed from put-call parity. Matlab code for computing option values is provided in Appendix E.

Armed with the quasi-closed form option-pricing formula in equation (20), we are ready to embark on a large-scale empirical investigation of the four models.

\subsection{Fitting Options and Returns}

From OptionMetrics we obtain Wednesday closing mid-quotes on SPX options data starting on 2 January 1996 and ending on 28 August 2013, which was the last date available at the time of writing.

We apply some commonly-used option data filters to the raw data. We restrict attention to out-of-the-money options with maturity between 15 and 180 calendar days. We omit contracts that do not satisfy well-known no-arbitrage conditions. We use only the six strikes with highest trading volume for each maturity quoted on Wednesdays. Finally, we convert puts to calls using put-call parity, so as to ease the computation and interpretation below.

Table 2 provides descriptive statistics of the resulting data set consisting of 21,283 options. The top panel shows the contracts sorted by moneyness defined using the Black-Scholes delta. The persistent "smile" pattern in implied volatility is readily apparent from the top panel. The middle panel sorts the contracts by maturity and shows that there is not a persistent 
maturity pattern in implied volatilities: the term structure of implied volatility is roughly flat, on average. The bottom panel sorts by the VIX level. Table 2 shows that roughly half the contracts have a delta above 0.6, a time-to-maturity between 30 and 90 days, and are recorded on days when the VIX is between 15 and 25.

Joint estimation is performed by following Trolle and Schwartz (2009), who assume that the vega-weighted option errors, $e_{j}$, are i.i.d. Gaussian. We can then define the option likelihood, $\ln L^{O}$, and the joint likelihood, $\ln L$, as follows:

$$
\begin{aligned}
V W R M S E & =\sqrt{\frac{1}{N} \sum_{j=1}^{N} e_{j}^{2}}=\sqrt{\frac{1}{N} \sum_{j=1}^{N}\left(\left(C_{j}^{M k t}-C_{j}^{M o d}\right) / B S V_{j}^{M k t}\right)^{2}} \\
\ln L^{O} & =-\frac{1}{2} \sum_{j=1}^{N}\left[\ln \left(V W R M S E^{2}\right)+e_{j}^{2} / V W R M S E^{2}\right] \\
\ln L & =\ln L^{P}+\ln L^{O},
\end{aligned}
$$

where $\ln L^{P}$ denotes the log-likelihood function of the physical process defined in equation (14). We then estimate all physical parameters and risk premia by maximizing the joint likelihood function, $\ln L$.

Table 3 contains the parameter estimates and log-likelihoods for our four models. We again calibrate the $\omega$ parameters by targeting the unconditional model variance to the sample variance of returns. As in Table 1, the physical parameters tend to be estimated precisely, whereas some of the risk premium parameters continue to be difficult to pin down. A sequential estimation procedure in which only risk premia are estimated from options may lead to more precise estimates. We leave this for future work.

The log-likelihoods reported in Table 3 are from joint estimation on returns and options for the GARCH model; from returns, RV and options for the RVM and RJM models; and from returns, $R B V, R J V$ and options for the BPRJM model. They are therefore not directly comparable.

The option errors at the bottom of the table, however, are comparable. They show that, in terms of implied volatility root mean squared error (IVRMSE), the RVM and RJM models offer a $12 \%$ improvement over the standard GARCH model. The BPRJM model offers a $17 \%$ improvement, which is quite impressive. The $V W R M S E$ metric is broadly consistent with the IVRMSE metric, again showing a $17 \%$ improvement of BPRJM over GARCH. 


\subsection{Exploring the Results}

In Table 4 we decompose the overall IVRMSE fit in Table 3 by moneyness, maturity and VIX level, following the layout of Table 2. The top panel of Table 4 shows that the BPRJM model performs the best in all but one moneyness category, namely deep out-of-the-money calls, where RVM is best. The BPRJM model performs particularly well for deep in-themoney calls (corresponding to deep out-of-the-money puts), which have proven notoriously difficult to fit in the literature. The middle panel of Table 4 shows that the BPRJM model performs the best in all maturity categories, including one virtual tie with the RJM model, namely for maturities between 30 and 60 days. The bottom panel shows that the BPRJM model is best in five of six VIX categories, and virtually tied in the sixth when VIX is between 15 and $20 \%$.

Altogether, Table 4 shows that the overall improvement in option fit by the BPRJM model evident in Table 3 is not due to any particular subset of the data set. The superior fit is obtained virtually everywhere.

Figure 7 reports the weekly time series of IVRMSE for at-the-money (ATM) options only. The figure is thus designed to reveal the models' ability to match the pattern of market volatility through time. Figure 7 shows that the RVM, RJM and BPRJM models are all much better than the GARCH model at capturing the dramatic dynamics in volatility unfolding during the 2008 financial crisis. It is indeed quite remarkable that the recent financial crisis does not appear as an outlier for the RVM, RJM and BPRJM models in Figure 7.

Figure 8 plots the model-implied risk-neutral higher moments over time for the 6 -month horizon. Note that the BPRJM model is able to generate higher skewness (middle panel) and excess kurtosis (lower panel) values than are the three other models. This feature of the model is likely a key driver in its success in fitting observed option prices, as is evident from Tables 3 and 4 .

The top panel of Figure 8 shows that the RVM, RJM and BPRJM models generate much higher 6-month risk-neutral volatility values than GARCH during the financial crisis in 2008. This is likely driving the at-the-money IVRMSE performance of these models evident from Figure 7.

\subsection{Option Error Specification}

The option-based objective function in (21) implicitly assumes that option errors are independent and identically distributed (iid) across contracts. In reality, option errors have complicated dependence structures that are likely a function of time, moneyness, time-tomaturity, and potentially also the level of the market and its volatility. Figure 7, which plots 
the weekly IVRMSE, indeed suggests that option errors are persistent across time. To study this issue further we plot in Figure 9 the autocorrelations of the weekly $V W R M S E$ series, which is ultimately part of the input into equation (21). As in Figure 7, we consider only ATM options in Figure 9. ${ }^{7}$ Figure 9 confirms that the option error magnitudes are indeed persistent over time.

The persistence in option errors implies that our maximum likelihood estimation procedure is not fully efficient. However, as long as the option errors are ergodic stationary (Hayashi, 2000, p. 465), we will still obtain consistent parameter estimates. Our estimates should thus be viewed as quasi maximum likelihood as opposed to fully maximum likelihood.

While we have not pursued them here, it is important to acknowledge that the literature has offered various approaches to account for the non-iid property of option errors. For example, Bates (2000) allows for heteroskedastic and autocorrelated errors in a Kalman filter approach. More recently, Kaeck and Alexander (2012) assume a multiplicative autoregressive error structure when estimating stochastic volatility models, and Kanniainen, Lin, and Yang (2014) assume additive autoregressive errors when estimating GARCH models.

Our focus is primarily on comparing different models and we conjecture that our comparisons are little affected by the quasi maximum likelihood estimation strategy that we apply uniformly across models. Nevertheless, specifying a more accurate error structure for the model we have suggested will likely lead to more efficient parameter estimates and therefore remains an important topic for future research.

\section{Summary and Conclusions}

Under very general conditions, the total quadratic variation of a stochastic price process can be decomposed into diffusive variation and squared jump variation. We have used this result to develop a new class of option valuation models in which the underlying asset price exhibits volatility and jump intensity dynamics. The first key feature of our model is that the volatility and jump intensity dynamics in the model are directly driven by model-free empirical measures of diffusive volatility and jump variation. Second, because the empirical measures are observed in discrete intervals, our option valuation model is cast in discrete time, allowing for straightforward estimation of the model. Third, our model belongs to the affine class, enabling us to derive the conditional characteristic function so that option values can be computed rapidly without relying on simulation methods. When estimated on S\&P500 index options, realized measures and returns, the new model performs well compared

\footnotetext{
${ }^{7}$ The autocorrelation plot using all options looks similar.
} 
with standard benchmarks.

Our analysis points to some interesting avenues for future research. First, a sequential estimation of physical parameters and then risk premia would be interesting. Second, implementing a more efficient estimation methodology would be of value, as discussed in Section 5.6. Third, several alternatives exist to the non-parametric measures of jumps explored in this paper. For example, Li (2013) employs hedging errors implied by delta-hedged positions in European-style options to identify jumps. Applying these alternative jump measures in our modelling framework could be useful. Fourth, we have allowed for measurement errors on some but not all variables. Extending our model in this direction would likely lead to even better empirical performance. Fifth, so far we have used only model-free physical measures of jumps and diffusive volatility. However, Du and Kapadia (2012) have recently proposed model-free risk-neutral counterparts to the realized bipower variation and realized jump variation measures we employ. Using the risk-neutral measures in our modelling framework may well lead to an even better fit. We leave these avenues open for future exploration. 


\section{References}

[1] Aït-Sahalia, Y., and J. Jacod (2012), Analyzing the Spectrum of Asset Returns: Jump and Volatility Components in High Frequency Data, Journal of Economic Literature, 50, 1007-1050.

[2] Andersen, T.G., and T. Bollerslev (1998), Answering the Skeptics: Yes, Standard Volatility Models Do Provide Accurate Forecasts, International Economic Review, 39, 885-905.

[3] Andersen, T.G., T. Bollerslev and F.X. Diebold (2007), Roughing it Up: Disentangling Continuous and Jump Components in Measuring, Modeling and Forecasting Asset Return Volatility, Review of Economics and Statistics, 89, 701-720.

[4] Andersen, T.G., T. Bollerslev, F.X. Diebold, and P. Labys (2003), Modeling and Forecasting Realized Volatility, Econometrica, 71, 579-625.

[5] Bakshi, G., C. Cao, and Z. Chen (1997), Empirical Performance of Alternative Option Pricing Models, Journal of Finance, 52, 2003-2050.

[6] Barndorff-Nielsen, O.E. and N. Shephard (2002), Econometric Analysis of Realised Volatility and Its Use in Estimating Stochastic Volatility Models, Journal of the Royal Statistical Society, Series B, 63, 253-280.

[7] Barndorff-Nielsen, O.E., and N. Shephard (2004), Power and Bipower Variation with Stochastic Volatility and Jumps, Journal of Financial Econometrics, 2, 1-37.

[8] Barndorff-Nielsen, O.E., and N. Shephard (2006), Econometrics of Testing for Jumps in Financial Economics Using Bipower Variation, Journal of Financial Econometrics, 4, $1-30$.

[9] Bates, D. (2000), Post-87 Crash Fears in S\&P500 Futures Options, Journal of Econometrics, 94, 181-238.

[10] Bates, D. (2012), U.S. Stock Market Crash Risk, 1926-2006, Journal of Financial Economics, 105, 229-259.

[11] Bollerslev, T., U. Kretschmer, C. Pigorsch, and G. Tauchen (2009), A Discrete-Time Model for Daily S\&P500 Returns and Realized Variations: Jumps and Leverage Effects, Journal of Econometrics, 150, 151-166. 
[12] Busch, T., B. J. Christensen, and M.O. Nielsen (2011), The Role of Implied Volatility in Forecasting Future Realized Volatility and Jumps in Foreign Exchange, Stock, and Bond Markets. Journal of Econometrics, 160, 48-57.

[13] Christoffersen, P., R. Elkamhi, B. Feunou, and K. Jacobs (2010), Option Valuation with Conditional Heteroskedasticity and Non-Normality, Review of Financial Studies, 23, 2139-2183.

[14] Christoffersen, P., B. Feunou, K. Jacobs and N. Meddahi (2014), The Economic Value of Realized Volatility: Using High-Frequency Returns for Option Valuation, Journal of Financial and Quantitative Analysis, 49, 663-697.

[15] Christoffersen, P., R. Goyenko, K. Jacobs, and M. Karoui, 2014, Illiquidity Premia in the Equity Options Market, Working Paper, University of Toronto.

[16] Christoffersen, P. , K. Jacobs, and C. Ornthanalai (2012), Dynamic Jump Intensities and Risk Premiums: Evidence from S\&P500 Returns and Options, Journal of Financial Economics, 106, 447-472.

[17] Corsi, F., N. Fusari, and D. La Vecchia (2013), Realizing Smiles: Options Pricing with Realized Volatility. Journal of Financial Economics, 107, 284-304.

[18] Du, J., and N. Kapadia (2012), Tail and Volatility Indices from Option Prices, Working Paper, University of Massachusetts, Amherst.

[19] Engle, R., and G. Gallo (2006), A Multiple Indicators Model for Volatility using Intradaily Data, Journal of Econometrics, 131, 3-27.

[20] Eraker, B. (2004), Do Stock Prices and Volatility Jump? Reconciling Evidence from Spot and Option Prices, Journal of Finance, 59, 1367-1403.

[21] Forsberg, L., and T. Bollerslev (2002), Bridging the Gap Between the Distribution of Realized (ECU) and ARCH Modelling (of the Euro): The GARCH-NIG Model, Journal of Applied Econometrics, 17, 535-548.

[22] Hansen, P., Z. Huang, and H. Shek (2012), Realized GARCH: A Joint Model for Returns and Realized Measures of Volatility, Journal of Applied Econometrics, 27, 877-906.

[23] Hansen, P., and A. Lunde (2005), A Realized Variance for the Whole Day Based on Intermittent High-Frequency Data, Journal of Financial Econometrics, 3, 525-554. 
[24] Hasanhodzic, J., and A. Lo (2011), Black's Leverage Effect Is Not Due To Leverage, Working Paper, MIT.

[25] Hayashi, F. (2000), Econometrics. Princeton University Press.

[26] Heston, S., and S. Nandi (2000), A Closed-Form GARCH Option Pricing Model, Review of Financial Studies, 13, 585-626.

[27] Huang, X., and G. Tauchen (2005), The Relative Contribution of Jumps to Total Price Variance, Journal of Financial Econometrics, 3, 456-499.

[28] Huang, J.-Z., and L. Wu (2004), Specification Analysis of Option Pricing Models based on Time-changed Levy Processes, Journal of Finance, 59, 1405-1439.

[29] Kaeck, A., and C. Alexander (2012), Volatility Dynamics for the S\&P 500: Further Evidence from Non-affine, Multi-factor Jump Diffusions, Journal of Banking and Finance, $36,3110-2121$.

[30] Kanniainen, J., B. Lin, and H. Yang (2014), Estimating and Using GARCH Models with VIX Data for Option Valuation, Journal of Banking and Finance, 43, 200-211.

[31] Li, J., 2013, Robust Estimation and Inference for Jumps in Noisy High Frequency Data: A Local-to-Continuity Theory for the Pre-averaging Method, Econometrica, 81, 6731693.

[32] Maheu, J., and T. McCurdy (2004), News Arrival, Jump Dynamics and Volatility Components for Individual Stock Returns, Journal of Finance, 59, 755-794.

[33] Ornthanalai, C. (2014), Lévy jump risk: Evidence from Options and Returns, Journal of Financial Economics, 112, 69-90.

[34] Santa-Clara, P., and S. Yan (2010), Crashes, Volatility and the Equity Premium: Lessons from S\&P500 Options, Review of Economics and Statistics, 92, 435-451.

[35] Shephard, N., and K. Sheppard (2010), Realising the Future: Forecasting with HighFrequency-Based Volatility (Heavy) models, Journal of Applied Econometrics, 25, 197231.

[36] Stentoft, L. (2008), Option Pricing Using Realized Volatility, CREATES Research Paper No. 2008-13. 
[37] Trolle, A., and E. Schwartz (2009), Unspanned Stochastic Volatility and the Pricing of Commodity Derivatives, Review of Financial Studies, 22, 4423-4461.

[38] Zhang, L., P. Mykland, and Y. Aït-Sahalia (2005), A Tale of Two Time Scales: Determining Integrated Volatility with Noisy High-Frequency Data, Journal of the American Statistical Association, 100, 1394-1411. 


\section{Appendix A: A Special Case of the Likelihood Function}

In this section, we compute a special case of the likelihood function used to fit the BPRJM model to the observed returns and RV only. Denote

$$
f_{t}\left(R_{t+1}, R V_{t+1}\right)=f_{t}\left(R_{t+1}, R B V_{t+1}+R J V_{t+1}\right) .
$$

Using the methodology from the general case, we have

$$
\begin{aligned}
f_{t}\left(R_{t+1}, R B V_{t+1}+R J V_{t+1}\right) & =\sum_{j=0}^{\infty} f_{t}\left(R_{t+1}, R B V_{t+1}+R J V_{t+1}, n_{t+1}=j\right) \\
& =\sum_{j=0}^{\infty} f_{t}\left(R_{t+1}, R B V_{t+1}+R J V_{t+1} \mid n_{t+1}=j\right) P_{t}\left[n_{t+1}=j\right],
\end{aligned}
$$

with

$$
\begin{gathered}
P_{t}\left[n_{t+1}=j\right]=\frac{e^{-h_{y, t}} h_{y, t}^{j}}{! j} \\
f_{t}\left(R_{t+1}, R B V_{t+1}+R J V_{t+1} \mid n_{t+1}=j\right)=\left\{\begin{array}{l}
f_{t}\left(R_{t+1}, R B V_{t+1}\right) \text { if } j=0 \\
\bar{f}_{t}(j)
\end{array}, \text { if } j>0\right.
\end{gathered},
$$

where

$$
\begin{gathered}
\bar{f}_{t}(j)=(2 \pi)^{-1}\left|\Omega_{t}^{(r, r v)}(0)\right|^{-1 / 2} \exp \left(-\frac{1}{2}\left(X_{t+1}^{(r, r v)}-\mu_{t}^{(r, r v)}(j)\right)^{\prime} \Omega_{t}^{(r, r v)}(j)^{-1}\left(X_{t+1}^{(r, r v)}-\mu_{t}^{(r, r v)}(j)\right)\right) \\
\mu_{t}^{(r, r v)}(j)=\left(\begin{array}{c}
r+\left(\lambda_{z}-\frac{1}{2}\right) h_{z, t}+\left(\lambda_{y}-\xi\right) h_{y, t}+\theta j \\
h_{z, t}+\left(\theta^{2}+\delta^{2}\right) j
\end{array}\right) \\
\Omega_{t}^{(r, r v)}(j)=\left[\begin{array}{ll}
h_{z, t}+\delta^{2} j & -2 \rho \gamma \sigma h_{z, t}+2 \theta \delta^{2} j \\
-2 \rho \gamma \sigma h_{z, t}+2 \theta \delta^{2} j & 2 \sigma^{2}\left(1+2 \gamma^{2} h_{z, t}\right)+2 \delta^{2}\left(\delta^{2}+2 \theta^{2}\right) j
\end{array}\right] .
\end{gathered}
$$

\section{Appendix B: Physical MGF for the BPRJM Model}

In this section, we derive the closed-form MGF for the BPRJM model under the physical measure. Using the vector notation $h_{t} \equiv\left(h_{z, t}, h_{y, t}\right)^{\prime}$ and further defining the coefficients 
$v \equiv\left(v_{z}, v_{y}\right)^{\prime}$, we can write the physical moment-generating function as $E_{t}\left[\exp \left(u R_{t+1}+v^{\prime} h_{t+1}\right)\right]=E_{t}\left[\exp \left(\begin{array}{c}u\left(r+\left(\lambda_{z}-\frac{1}{2}\right) h_{z, t}+\left(\lambda_{y}-\xi\right) h_{y, t}+z_{t+1}+y_{t+1}\right) \\ +v^{\prime}\left(\omega+b h_{t}+a R V M_{t+1}\right)\end{array}\right)\right]=$
$\exp \left(u\left(r+\left(\lambda_{z}-\frac{1}{2}\right) h_{z, t}+\left(\lambda_{y}-\xi\right) h_{y, t}\right)+v^{\prime}\left(\omega+b h_{t}\right)\right) E_{t}\left[\exp \left(u\left(z_{t+1}+y_{t+1}\right)+v^{\prime} a R V M_{t+1}\right)\right]$

We further define

$$
v^{\prime} a=\left(v_{z}, v_{y}\right)\left(\begin{array}{cc}
a_{z} & a_{z, y} \\
a_{y, z} & a_{y}
\end{array}\right)=\left(v_{z} a_{z}+v_{y} a_{y, z}, v_{z} a_{z, y}+v_{y} a_{y}\right) \equiv\left(v_{1}, v_{2}\right) .
$$

Then, we can write

$$
\begin{aligned}
& E_{t}\left[\exp \left(u\left(z_{t+1}+y_{t+1}\right)+v^{\prime} a R V M_{t+1}\right)\right] \\
= & E_{t}\left[\exp \left(u\left(z_{t+1}+y_{t+1}\right)+v_{1} R B V_{t+1}+v_{2} R J V_{t+1}\right)\right] \\
= & \exp \left(v_{1}\left(h_{z, t}-\sigma\left(1+\gamma^{2} h_{z, t}\right)\right)\right) E_{t}\left[\exp \left(u \sqrt{h_{z, t}} \varepsilon_{1, t+1}+v_{1} \sigma\left(\varepsilon_{2, t+1}-\gamma \sqrt{h_{z, t}}\right)^{2}\right)\right] \times \\
& E_{t}\left[\exp \left(\sum_{j=0}^{n_{t+1}} u x_{t+1}^{j}+v_{2}\left(x_{t+1}^{j}\right)^{2}\right)\right],
\end{aligned}
$$

where the expectations can be computed explicitly as

$$
\begin{aligned}
& E_{t}\left[\exp \left(u \sqrt{h_{z, t}} \varepsilon_{1, t+1}+v_{1} \sigma\left(\varepsilon_{2, t+1}-\gamma \sqrt{h_{z, t}}\right)^{2}\right)\right] \\
= & \exp \left(-\frac{1}{2} \ln \left(1-2 \sigma v_{1}\right)+\left(v_{1} \sigma \gamma^{2}+\frac{1}{2}\left(1-\rho^{2}\right) u^{2}+\frac{\left(u \rho-2 \sigma v_{1} \gamma\right)^{2}}{2\left(1-2 \sigma v_{1}\right)}\right) h_{z, t}\right),
\end{aligned}
$$

and

$$
\begin{gathered}
E_{t}\left[\exp \left(\sum_{j=0}^{n_{t+1}} u x_{t+1}^{j}+v_{2}\left(x_{t+1}^{j}\right)^{2}\right)\right]=E_{t}\left[E_{t}\left[\exp \left(\sum_{j=0}^{n_{t+1}} u x_{t+1}^{j}+v_{2}\left(x_{t+1}^{j}\right)^{2}\right) \mid n_{t+1}\right]\right] \\
E_{t}\left[\exp \left(\sum_{j=0}^{n_{t+1}} u x_{t+1}^{j}+v_{2}\left(x_{t+1}^{j}\right)^{2}\right) \mid n_{t+1}\right]=\exp \left(v_{3} n_{t+1}\right),
\end{gathered}
$$

where

$$
v_{3}=-\frac{1}{2} \ln \left(1-2 v_{2} \delta^{2}\right)+u \theta+v_{2} \theta^{2}+\frac{\left(u+2 \theta v_{2}\right)^{2} \delta^{2}}{2\left(1-2 v_{2} \delta^{2}\right)} .
$$


Hence

$$
E_{t}\left[\exp \left(\sum_{j=0}^{n_{t+1}} u x_{t+1}^{j}+v_{2}\left(x_{t+1}^{j}\right)^{2}\right)\right]=E_{t}\left[\exp \left(v_{3} n_{t+1}\right)\right]=\exp \left(h_{y, t}\left(e^{v_{3}}-1\right)\right) .
$$

Therefore, we have the following expression

$$
E_{t}\left[\exp \left(u\left(z_{t+1}+y_{t+1}\right)+v^{\prime} a R V M_{t+1}\right)\right]=\exp \left(\begin{array}{c}
v_{1}\left(h_{z, t}-\sigma\left(1+\gamma^{2} h_{z, t}\right)\right)-\frac{1}{2} \ln \left(1-2 \sigma v_{1}\right) \\
+\left(v_{1} \sigma \gamma^{2}+\frac{1}{2}\left(1-\rho^{2}\right) u^{2}+\frac{\left(u \rho-2 \sigma v_{1} \gamma\right)^{2}}{2\left(1-2 \sigma v_{1}\right)}\right) h_{z, t} \\
+\left(e^{v_{3}}-1\right) h_{y, t}
\end{array}\right) .
$$

Substituting the above back into the original MGF, we get

$$
\begin{aligned}
E_{t}\left[\exp \left(u R_{t+1}+v^{\prime} h_{t+1}\right)\right] & =\exp \left(\begin{array}{c}
u\left(r+\left(\lambda_{z}-\frac{1}{2}\right) h_{z, t}+\left(\lambda_{y}-\xi\right) h_{y, t}\right)+v^{\prime}\left(\omega+b h_{t}\right) \\
+v_{1}\left(h_{z, t}-\sigma\left(1+\gamma^{2} h_{z, t}\right)\right)-\frac{1}{2} \ln \left(1-2 \sigma v_{1}\right) \\
+\left(v_{1} \sigma \gamma^{2}+\frac{1}{2}\left(1-\rho^{2}\right) u^{2}+\frac{\left(u \rho-2 \sigma v_{1}\right)^{2}}{2\left(1-2 \sigma v_{1}\right)}\right) h_{z, t}+\left(e^{v_{3}}-1\right) h_{y, t}
\end{array}\right) \\
& \equiv \exp \left(A(u, v)^{\prime} h_{t}+B(u, v)\right),
\end{aligned}
$$

which shows that the physical one-step-ahead moment-generating function is exponentially affine.

We conjecture that the multi-step moment-generating function is also of the affine form. First, define

$$
\begin{aligned}
\Psi_{t, t+M}(u) & =E_{t}\left[\exp \left(u \sum_{j=1}^{M} R_{t+j}\right)\right] \\
& =\exp \left(C(u, M)^{\prime} h_{t}+D(u, M)\right) .
\end{aligned}
$$

From this we can compute

$$
\begin{aligned}
\Psi_{t, t+M+1}(u) & =E_{t}\left[\exp \left(u \sum_{j=1}^{M} R_{t+j}\right)\right]=E_{t}\left[E_{t+1}\left[\exp \left(u \sum_{j=1}^{M} R_{t+j}\right)\right]\right] \\
& =E_{t}\left[\exp \left(u R_{t+1}\right) E_{t+1}\left[\exp \left(u \sum_{j=2}^{M} R_{t+j}\right)\right]\right] \\
& =E_{t}\left[\exp \left(u R_{t+1}+C(u, M)^{\prime} h_{t+1}+D(u, M)\right)\right] \\
& =\exp \left(A(u, C(u, M))^{\prime} h_{t}+B(u, C(u, M))+D(u, M)\right),
\end{aligned}
$$


which yields the following recursive relationship:

$$
\begin{aligned}
& C(u, M+1)=A(u, C(u, M)) \\
& D(u, M+1)=B(u, C(u, M))+D(u, M),
\end{aligned}
$$

including the following initial conditions:

$$
\begin{aligned}
& C(u, 1)=A(u, \mathbf{0}) \\
& D(u, 1)=B(u, \mathbf{0})
\end{aligned}
$$

where $A$ and $C$ are 2-by-1 vector-valued functions.

\section{Appendix C: Risk Neutralization of the BPRJM Model}

In this appendix, we derive the risk neutralization of the BPRJM model. We assume an exponential pricing kernel of the following form:

$$
\begin{aligned}
\zeta_{t+1} & =\frac{M_{t+1}}{E_{t}\left[M_{t+1}\right]} \equiv \frac{\exp \left(\nu_{1, t} \varepsilon_{1, t+1}+\nu_{2, t} \varepsilon_{2, t+1}+\nu_{3, t} \sum_{j=0}^{n_{t+1}} x_{t+1}^{j}\right)}{E_{t}\left[\exp \left(\nu_{1, t} \varepsilon_{1, t+1}+\nu_{2, t} \varepsilon_{2, t+1}+\nu_{3, t} \sum_{j=0}^{n_{t+1}} x_{t+1}^{j}\right)\right]} \\
& =\exp \left(\begin{array}{c}
\nu_{1, t} \varepsilon_{1, t+1}+\nu_{2, t} \varepsilon_{2, t+1}+\nu_{3, t} \sum_{j=0}^{n_{t+1}} x_{t+1}^{j} \\
\left.-\frac{1}{2} \nu_{1, t}^{2}-\frac{1}{2} \nu_{2, t}^{2}-\rho \nu_{1, t} \nu_{2, t}-\left(e^{\theta \nu_{3, t}+\frac{1}{2} \delta^{2} \nu_{3, t}^{2}}-1\right) h_{y, t}\right)
\end{array}\right.
\end{aligned}
$$

We need to impose the no-arbitrage condition

$$
E_{t}^{Q}\left[\exp \left(R_{t+1}\right)\right] \equiv E_{t}\left[\zeta_{t+1} \exp \left(R_{t+1}\right)\right]=\exp (r)
$$


where

$$
\begin{aligned}
& E_{t}\left[\zeta_{t+1} \exp \left(R_{t+1}\right)\right]=E_{t}\left[\zeta_{t+1} \exp \left(r+\left(\lambda_{z}-\frac{1}{2}\right) h_{z, t}+\left(\lambda_{y}-\xi\right) h_{y, t}+z_{t+1}+y_{t+1}\right)\right] \\
& =\exp \left(\begin{array}{c}
r+\left(\lambda_{z}-\frac{1}{2}\right) h_{z, t}+\left(\lambda_{y}-\xi\right) h_{y, t} \\
-\frac{1}{2} \nu_{1, t}^{2}-\frac{1}{2} \nu_{2, t}^{2}-\rho \nu_{1, t} \nu_{2, t}-\left(e^{\theta \nu_{3, t}+\frac{1}{2} \delta^{2} \nu_{3, t}^{2}}-1\right) h_{y, t} \\
+\frac{1}{2}\left(\nu_{1, t}+\sqrt{h_{z, t}}\right)^{2}+\frac{1}{2} \nu_{2, t}^{2}+\rho\left(\nu_{1, t}+\sqrt{h_{z, t}}\right) \nu_{2, t} \\
+\left(e^{\theta\left(\nu_{3, t}+1\right)+\frac{1}{2} \delta^{2}\left(\nu_{3, t}+1\right)^{2}}-1\right) h_{y, t}
\end{array}\right) \\
& =\exp \left(\begin{array}{c}
r+\lambda_{z} h_{z, t}+\left(\lambda_{y}-\xi\right) h_{y, t} \\
e^{\theta \nu_{3, t}+\frac{1}{2} \delta^{2} \nu_{3, t}^{2}}\left(e^{\theta+\frac{1}{2} \delta^{2}+\delta^{2} \nu_{3, t}}-1\right) h_{y, t} \\
+\nu_{1, t} \sqrt{h_{z, t}}+\rho \sqrt{h_{z, t}} \nu_{2, t}
\end{array}\right) .
\end{aligned}
$$

Setting this expression equal to the risk-free rate and taking logs yields the condition

$$
\lambda_{z} h_{z, t}+\left(\lambda_{y}-\xi\right) h_{y, t}+\nu_{1, t} \sqrt{h_{z, t}}+\rho \sqrt{h_{z, t}} \nu_{2, t}+e^{\theta \nu_{3, t}+\frac{1}{2} \delta^{2} \nu_{3, t}^{2}}\left(e^{\theta+\frac{1}{2} \delta^{2}+\delta^{2} \nu_{3, t}}-1\right) h_{y, t}=0
$$

In order to determine the form of the risk-neutral distribution of the shocks, we consider the moment-generating function

$$
E_{t}^{Q}\left[\exp \left(u_{1} \varepsilon_{1, t+1}+u_{2} \varepsilon_{2, t+1}+u_{3} y_{t+1}\right)\right]=\exp \left(\begin{array}{c}
u_{1}\left(\nu_{1, t}+\rho \nu_{2, t}\right)+u_{2}\left(\nu_{2, t}+\rho \nu_{1, t}\right)+\frac{u_{1}^{2}}{2}+\frac{u_{2}^{2}}{2}+\rho u_{1} u_{2} \\
+\left(e^{\left(\theta+\delta^{2} \nu_{3, t}\right) u_{3}+\frac{1}{2} \delta^{2} u_{3}^{2}}-1\right) e^{\theta \nu_{3, t}+\frac{1}{2} \delta^{2} \nu_{3, t}^{2}} h_{y, t}
\end{array}\right)
$$

In order to obtain an affine model under the $Q$ measure, we set $\nu_{3, t}$ to a constant, i.e. $\nu_{3, t}=\nu_{3}$. Under the $Q$ measure we have

$$
\begin{aligned}
\varepsilon_{1, t+1}^{*} & =\varepsilon_{1, t+1}-\left(\nu_{1, t}+\rho \nu_{2, t}\right) ; \quad \varepsilon_{1, t+1}^{*} \stackrel{i i d^{Q}}{\sim} N(0,1) \\
\varepsilon_{2, t+1}^{*} & =\varepsilon_{2, t+1}-\left(\nu_{2, t}+\rho \nu_{1, t}\right) ; \quad \varepsilon_{2, t+1}^{*} \stackrel{i i d^{Q}}{\sim} N(0,1) \\
y_{t+1} & =\sum_{j=0}^{n_{t+1}} x_{t+1}^{j} ; \quad x_{t+1}^{j} \stackrel{i i d^{Q}}{\sim} N\left(\theta+\delta^{2} \nu_{3}, \delta^{2}\right) ; \quad n_{t+1} \mid I_{t} \sim^{Q} \operatorname{Poisson}\left(e^{\theta \nu_{3}+\frac{1}{2} \delta^{2} \nu_{3}^{2}} h_{y, t}\right) .
\end{aligned}
$$

We thus see that, under the $Q$ measure, $\varepsilon_{1, t+1}^{*}$ and $\varepsilon_{2, t+1}^{*}$ follow a bivariate standard normal distribution with correlation $\rho$. 
The realized bipower variation equation can be written as follows:

$$
\begin{aligned}
R B V_{t+1} & =h_{z, t}+\sigma\left[\left(\varepsilon_{2, t+1}-\gamma \sqrt{h_{z, t}}\right)^{2}-\left(1+\gamma^{2} h_{z, t}\right)\right] \\
& =h_{z, t}+\sigma\left[\left(\varepsilon_{2, t+1}^{*}+\nu_{2, t}+\rho \nu_{1, t}-\gamma \sqrt{h_{z, t}}\right)^{2}-\left(1+\gamma^{2} h_{z, t}\right)\right] .
\end{aligned}
$$

In order to ensure that the model is affine under $Q$, it is necessary and sufficient to fix

$$
\nu_{2, t}+\rho \nu_{1, t}-\gamma \sqrt{h_{z, t}}=-\gamma^{*} \sqrt{h_{z, t}},
$$

which yields the condition

$$
\nu_{2, t}=\left(\gamma-\gamma^{*}\right) \sqrt{h_{z, t}}-\rho \nu_{1, t}
$$

Using the no-arbitrage condition above implies that

$\nu_{1, t} \sqrt{h_{z, t}}+\rho \sqrt{h_{z, t}}\left(\left(\gamma-\gamma^{*}\right) \sqrt{h_{z, t}}-\rho \nu_{1, t}\right)=-\lambda_{z} h_{z, t}-\left(\lambda_{y}-\xi\right) h_{y, t}-e^{\theta \nu_{3}+\frac{1}{2} \delta^{2} \nu_{3}^{2}}\left(e^{\theta+\frac{1}{2} \delta^{2}+\delta^{2} \nu_{3}}-1\right) h_{y, t}$.

Thus we have

$$
\begin{gathered}
\nu_{1, t}=\frac{\left(\rho\left(\gamma^{*}-\gamma\right)-\lambda_{z}\right) h_{z, t}-\left(\lambda_{y}-\xi+e^{\theta \nu_{3}+\frac{1}{2} \delta^{2} \nu_{3}^{2}}\left(e^{\theta+\frac{1}{2} \delta^{2}+\delta^{2} \nu_{3}}-1\right)\right) h_{y, t}}{\left(1-\rho^{2}\right) \sqrt{h_{z, t}}} \\
\left(\nu_{1, t}+\rho \nu_{2, t}\right) \sqrt{h_{z, t}}=-\lambda_{z} h_{z, t}-\left(\left(\lambda_{y}-\xi\right)+e^{\theta \nu_{3}+\frac{1}{2} \delta^{2} \nu_{3}^{2}}\left(e^{\theta+\frac{1}{2} \delta^{2}+\delta^{2} \nu_{3}}-1\right)\right) h_{y, t} .
\end{gathered}
$$

Next we can rewrite the returns equation under the risk-neutral measure as follows:

$$
\begin{aligned}
R_{t+1} & \equiv \log \left(\frac{S_{t+1}}{S_{t}}\right)=r+\left(\lambda_{z}-\frac{1}{2}\right) h_{z, t}+\left(\lambda_{y}-\xi\right) h_{y, t}+z_{t+1}+y_{t+1} \\
& =r-\frac{1}{2} h_{z, t}-e^{\theta \nu_{3}+\frac{1}{2} \delta^{2} \nu_{3}^{2}}\left(e^{\theta+\frac{1}{2} \delta^{2}+\delta^{2} \nu_{3}}-1\right) h_{y, t}+\sqrt{h_{z, t}} \varepsilon_{1, t+1}^{*} \\
& =r-\frac{1}{2} h_{z, t}-\xi^{*} h_{y, t}^{*}+\sqrt{h_{z, t}} \varepsilon_{1, t+1}^{*}+y_{t+1} .
\end{aligned}
$$


Hence under the risk-neutral measure, we have

$$
\begin{aligned}
R_{t+1} & \equiv \log \left(\frac{S_{t+1}}{S_{t}}\right)=r-\frac{1}{2} h_{z, t}-\xi^{*} h_{y, t}^{*}+\sqrt{h_{z, t}} \varepsilon_{1, t+1}^{*}+y_{t+1} \\
y_{t+1} & =\sum_{j=0}^{n_{t+1}} x_{t+1}^{j} ; \quad x_{t+1}^{j} \sim^{Q} \operatorname{iidN}\left(\theta^{*}, \delta^{2}\right) ; \quad n_{t+1} \mid I_{t} \sim^{Q} \operatorname{Poisson}\left(h_{y, t}^{*}\right) \\
R B V_{t+1} & =h_{z, t}+\left(\left(\gamma^{*}\right)^{2}-\gamma^{2}\right) h_{z, t}+\sigma\left[\left(\varepsilon_{2, t+1}^{*}-\gamma^{*} \sqrt{h_{z, t}}\right)^{2}-\left(1+\left(\gamma^{*}\right)^{2} h_{z, t}\right)\right] \\
R J V_{t+1} & =\sum_{j=0}^{n_{t+1}}\left(x_{t+1}^{j}\right)^{2},
\end{aligned}
$$

with the following parameter mapping:

$$
\begin{aligned}
h_{y, t}^{*} & =e^{\theta \nu_{3}+\frac{1}{2} \delta^{2} \nu_{3}^{2}} h_{y, t} \\
\theta^{*} & =\theta+\delta^{2} \nu_{3}, \quad \xi^{*}=e^{\theta^{*}+\frac{1}{2} \delta^{2}}-1 .
\end{aligned}
$$

\section{Appendix D: Risk Neutralization of the RJM Model}

In this appendix, we derive the risk neutralization of the RJM model. We use the following exponential form of the pricing kernel to ensure that the affine structure is preserved under the risk-neutral measure:

$$
\zeta_{t+1}=\frac{M_{t+1}}{E_{t}\left[M_{t+1}\right]} \equiv \frac{\exp \left(\nu_{1} \sum_{j=1}^{n_{t+1}} x_{t+1}^{j}+\nu_{2} \sum_{j=1}^{n_{t+1}}\left(x_{t+1}^{j}\right)^{2}+\nu_{3} n_{t+1}\right)}{E_{t}\left[\exp \left(\nu_{1} \sum_{j=1}^{n_{t+1}} x_{t+1}^{j}+\nu_{2} \sum_{j=1}^{n_{t+1}}\left(x_{t+1}^{j}\right)^{2}+\nu_{3} n_{t+1}\right)\right]},
$$


which can be written as

$$
\begin{aligned}
& E_{t}\left[\exp \left(\nu_{1} \sum_{j=1}^{n_{t+1}} x_{t+1}^{j}+\nu_{2} \sum_{j=1}^{n_{t+1}}\left(x_{t+1}^{j}\right)^{2}+\nu_{3} n_{t+1}\right)\right] \\
= & E_{t}\left[E_{t}\left[\exp \left(\nu_{1} \sum_{j=1}^{n_{t+1}} x_{t+1}^{j}+\nu_{2} \sum_{j=1}^{n_{t+1}}\left(x_{t+1}^{j}\right)^{2}+\nu_{3} n_{t+1}\right) \mid n_{t+1}\right]\right] \\
= & E_{t}\left[\exp \left(\nu_{3} n_{t+1}\right) E_{t}\left[\exp \left(\sum_{j=1}^{n_{t+1}}\left(\nu_{1} x_{t+1}^{j}+\nu_{2}\left(x_{t+1}^{j}\right)^{2}\right)\right) \mid n_{t+1}\right]\right] \\
= & E_{t}\left[\exp \left(\nu_{3} n_{t+1}\right) \prod_{j=1}^{n_{t+1}} E_{t}\left[\exp \left(\nu_{1} x_{t+1}^{j}+\nu_{2}\left(x_{t+1}^{j}\right)^{2}\right) \mid n_{t+1}\right]\right] \\
= & E_{t}\left[\exp \left(\nu_{3} n_{t+1}\right)\left(E_{t}\left[\exp \left(\nu_{1} x_{t+1}^{j}+\nu_{2}\left(x_{t+1}^{j}\right)^{2}\right)\right]\right)^{n_{t+1}}\right] \\
= & E_{t}\left[\exp \left(\nu_{4} n_{t+1}\right)\right]
\end{aligned}
$$

using the definition

$$
\nu_{4}=-\frac{1}{2} \ln \left(1-2 \nu_{2} \delta^{2}\right)+\nu_{1} \theta+\nu_{2} \theta^{2}+\frac{\left(\nu_{1}+2 \theta \nu_{2}\right)^{2} \delta^{2}}{2\left(1-2 \nu_{2} \delta^{2}\right)}+\nu_{3} .
$$

Hence we have

$$
\zeta_{t+1}=\exp \left(\nu_{1} \sum_{j=1}^{n_{t+1}} x_{t+1}^{j}+\nu_{2} \sum_{j=1}^{n_{t+1}}\left(x_{t+1}^{j}\right)^{2}-\nu_{4}-\left(e^{\nu_{4}}-1\right) h_{y, t}\right)
$$

We need to impose the no-arbitrage condition

$$
E_{t}^{Q}\left[\exp \left(R_{t+1}\right)\right] \equiv E_{t}\left[\zeta_{t+1} \exp \left(R_{t+1}\right)\right]=\exp (r),
$$

which gives us the following parameter restriction:

$$
\begin{aligned}
E_{t}\left[\zeta_{t+1} \exp \left(R_{t+1}\right)\right] & =E_{t}\left[\zeta_{t+1} \exp \left(\bar{r}+\left(\lambda_{y}-\xi\right) h_{y, t}+\sum_{j=1}^{n_{t+1}} x_{t+1}^{j}\right)\right] \\
& =E_{t}\left[\exp \left(\begin{array}{c}
\bar{r}+\left(\lambda_{y}-\xi\right) h_{y, t}+\left(1+\nu_{1}\right) \sum_{j=1}^{n_{t+1}} x_{t+1}^{j}+ \\
\nu_{2} \sum_{j=1}^{n_{t+1}}\left(x_{t+1}^{j}\right)^{2}-\nu_{4}-\left(e^{\nu_{4}}-1\right) h_{y, t}
\end{array}\right)\right] \\
& =\exp \left(\bar{r}+\nu_{5}-\nu_{4}+\left(\lambda_{y}-\xi+e^{\nu_{5}}-e^{\nu_{4}}\right) h_{y, t}\right),
\end{aligned}
$$


with

$$
\nu_{5}=-\frac{1}{2} \ln \left(1-2 \nu_{2} \delta^{2}\right)+\left(1+\nu_{1}\right) \theta+\nu_{2} \theta^{2}+\frac{\left(1+\nu_{1}+2 \theta \nu_{2}\right)^{2} \delta^{2}}{2\left(1-2 \nu_{2} \delta^{2}\right)}+\nu_{3} .
$$

Hence, the following relationships need to hold:

$$
\begin{aligned}
\nu_{5}-\nu_{4} & =\theta+\frac{\delta^{2}}{2} \\
e^{\nu_{5}}-e^{\nu_{4}} & =\xi-\lambda_{y} .
\end{aligned}
$$

Thus

$$
\begin{aligned}
e^{\nu_{5}}-e^{\nu_{4}} & =e^{\nu_{4}}\left(e^{\nu_{5}-\nu_{4}}-1\right) \\
& =e^{\nu_{4}}\left(e^{\theta+\frac{\delta^{2}}{2}}-1\right) \\
& =e^{\nu_{4}} \xi \\
e^{\nu_{4}} & =\frac{\xi-\lambda_{y}}{\xi},
\end{aligned}
$$

and

$$
\begin{gathered}
\nu_{4}=\ln \left(1-\frac{\lambda_{y}}{\xi}\right) \\
\nu_{5}-\nu_{4}=\theta+\frac{\left[\left(1+\nu_{1}+2 \theta \nu_{2}\right)^{2}-\left(\nu_{1}+2 \theta \nu_{2}\right)^{2}\right] \delta^{2}}{2\left(1-2 \nu_{2} \delta^{2}\right)} \\
=\theta+\frac{\left(1+2 \nu_{1}+4 \theta \nu_{2}\right) \delta^{2}}{2\left(1-2 \nu_{2} \delta^{2}\right)} \\
\nu_{5}-\nu_{4}=\theta+\frac{\delta^{2}}{2},
\end{gathered}
$$

which implies that

$$
\theta+\frac{\delta^{2}}{2}=\theta+\frac{\left(1+2 \nu_{1}+4 \theta \nu_{2}\right) \delta^{2}}{2\left(1-2 \nu_{2} \delta^{2}\right)}
$$

Hence

$$
1+2 \nu_{1}+4 \theta \nu_{2}=1-2 \nu_{2} \delta^{2}
$$

which can be written as

$$
\nu_{1}=-\left(\delta^{2}+2 \theta\right) \nu_{2},
$$


and

$$
\begin{aligned}
\nu_{3} & =\nu_{4}-\left(-\frac{1}{2} \ln \left(1-2 \nu_{2} \delta^{2}\right)+\nu_{1} \theta+\nu_{2} \theta^{2}+\frac{\left(\nu_{1}+2 \theta \nu_{2}\right)^{2} \delta^{2}}{2\left(1-2 \nu_{2} \delta^{2}\right)}\right) \\
& =\ln \left(1-\frac{\lambda_{y}}{\xi}\right)+\frac{1}{2} \ln \left(1-2 \nu_{2} \delta^{2}\right)+\theta\left(\delta^{2}+\theta\right) \nu_{2}-\frac{\delta^{6} \nu_{2}^{2}}{2\left(1-2 \nu_{2} \delta^{2}\right)} .
\end{aligned}
$$

To determine the risk-neutral distribution of the shocks, we consider

$$
\begin{aligned}
E_{t}^{Q}\left[\exp \left(u n_{t+1}\right)\right] & =E_{t}\left[\exp \left(u n_{t+1}+\nu_{1} \sum_{j=1}^{n_{t+1}} x_{t+1}^{j}+\nu_{2} \sum_{j=1}^{n_{t+1}}\left(x_{t+1}^{j}\right)^{2}+\nu_{3} n_{t+1}-\nu_{4}-\left(e^{\nu_{4}}-1\right) h_{y, t}\right)\right] \\
& =\exp \left(-\nu_{4}-\left(e^{\nu_{4}}-1\right) h_{y, t}\right) E_{t}\left[\exp \left(v n_{t+1}\right)\right] \\
& =\exp \left(-\nu_{4}-\left(e^{\nu_{4}}-1\right) h_{y, t}+v+\left(e^{v}-1\right) h_{y, t}\right) \\
& =\exp \left(v-\nu_{4}+\left(e^{v}-e^{\nu_{4}}\right) h_{y, t}\right)=\exp \left(v-\nu_{4}+e^{\nu_{4}}\left(e^{v-\nu_{4}}-1\right) h_{y, t}\right)
\end{aligned}
$$

with

$$
\begin{gathered}
v=-\frac{1}{2} \ln \left(1-2 \nu_{2} \delta^{2}\right)+\nu_{1} \theta+\nu_{2} \theta^{2}+\frac{\left(\nu_{1}+2 \theta \nu_{2}\right)^{2} \delta^{2}}{2\left(1-2 \nu_{2} \delta^{2}\right)}+u+\nu_{3} \\
v-\nu_{4}=u .
\end{gathered}
$$

Hence

$$
\begin{gathered}
E_{t}^{Q}\left[\exp \left(u n_{t+1}\right)\right]=\exp \left(u+\left(e^{u}-1\right)\left(1-\frac{\lambda_{y}}{\xi}\right) h_{y, t}\right) \\
n_{t+1}=n_{t+1}^{*}+1,
\end{gathered}
$$

where

$$
\begin{gathered}
n_{t+1}^{*} \mid I_{t}^{\sim Q} \operatorname{Poisson}\left(h_{y, t}^{*}\right) \\
h_{y, t}^{*}=\left(1-\frac{\lambda_{y}}{\xi}\right) h_{y, t} .
\end{gathered}
$$

Next we compute the conditional moment-generating function of individual jumps under the risk-neutral measure:

$$
\begin{aligned}
E_{t}^{Q}\left[\exp \left(u x_{t+1}^{j_{0}}\right)\right]= & E_{t}\left[\exp \left(\begin{array}{c}
u x_{t+1}^{j_{0}}+\nu_{1} \sum_{j=1}^{n_{t+1}} x_{t+1}^{j}+\nu_{2} \sum_{j=1}^{n_{t+1}}\left(x_{t+1}^{j}\right)^{2}+ \\
\nu_{3}\left(n_{t+1}-1\right)+\nu_{3}-\nu_{4}-\left(e^{\nu_{4}}-1\right) h_{y, t}
\end{array}\right)\right] \\
= & \exp \left(\nu_{3}-\nu_{4}-\left(e^{\nu_{4}}-1\right) h_{y, t}\right) E_{t}\left[\exp \left(v_{4}\left(n_{t+1}-1\right)\right)\right] \times \\
& \exp \left(-\frac{1}{2} \ln \left(1-2 \nu_{2} \delta^{2}\right)+\left(u+\nu_{1}\right) \theta+\nu_{2} \theta^{2}+\frac{\left(u+\nu_{1}+2 \theta \nu_{2}\right)^{2} \delta^{2}}{2\left(1-2 \nu_{2} \delta^{2}\right)}\right)
\end{aligned}
$$




$$
\begin{aligned}
E_{t}^{Q}\left[\exp \left(u x_{t+1}^{j_{0}}\right)\right] & =\exp \left(\begin{array}{r}
-\frac{1}{2} \ln \left(1-2 \nu_{2} \delta^{2}\right)+\left(u+\nu_{1}\right) \theta+\nu_{2} \theta^{2}+\frac{\left(u+\nu_{1}+2 \theta \nu_{2}\right)^{2} \delta^{2}}{2\left(1-2 \nu_{2} \delta^{2}\right)} \\
+\frac{1}{2} \ln \left(1-2 \nu_{2} \delta^{2}\right)-\nu_{1} \theta-\nu_{2} \theta^{2}-\frac{\left(\nu_{1}+2 \theta \nu_{2}\right)^{2} \delta^{2}}{2\left(1-2 \nu_{2} \delta^{2}\right)}
\end{array}\right) \\
& \equiv \exp \left(u \theta^{*}+\frac{\left(\delta^{*}\right)^{2}}{2} u^{2}\right)
\end{aligned}
$$

with the following parameter mappings:

$$
\begin{aligned}
\theta^{*} & =\theta+\frac{\left(\nu_{1}+2 \theta \nu_{2}\right) \delta^{2}}{\left(1-2 \nu_{2} \delta^{2}\right)}=\theta-\frac{\nu_{2} \delta^{4}}{\left(1-2 \nu_{2} \delta^{2}\right)} \\
\left(\delta^{*}\right)^{2} & =\frac{\delta^{2}}{1-2 \nu_{2} \delta^{2}}
\end{aligned}
$$

Thus we can rewrite the return equation under the risk-neutral measure as

$$
\begin{aligned}
R_{t+1} & =\bar{r}+\left(\lambda_{y}-\xi\right) h_{y, t}+y_{t+1} \\
\bar{r} & =r-\theta-\frac{\delta^{2}}{2} \\
\xi & =e^{\theta+\frac{1}{2} \delta^{2}}-1 \\
y_{t+1} & =\sum_{j=1}^{n_{t+1}} x_{t+1}^{j}, \quad x_{t+1}^{j} \sim Q_{i i d N}\left(\theta^{*},\left(\delta^{*}\right)^{2}\right) \\
Q\left[n_{t+1}\right. & \left.=k \mid I_{t}\right]=\frac{e^{-h_{y, t}^{*}\left(h_{y, t}^{*}\right)^{k-1}}}{!(k-1)} \\
h_{y, t}^{*} & =\left(1-\frac{\lambda_{y}}{\xi}\right) h_{y, t} \\
R V_{t+1} & =\sum_{j=1}^{n_{t+1}}\left(x_{t+1}^{j}\right)^{2}-\theta^{2} \\
h_{y, t+1}= & \omega_{y}+b_{y} h_{y, t}+a_{y} R V_{t+1} \\
\theta^{*}= & \theta+\frac{\left(\nu_{1}+2 \theta \nu_{2}\right) \delta^{2}}{\left(1-2 \nu_{2} \delta^{2}\right)}, \quad\left(\delta^{*}\right)^{2}=\frac{\delta^{2}}{1-2 \nu_{2} \delta^{2}}
\end{aligned}
$$

where $\nu_{2}$ is a parameter to be estimated.

\section{Appendix E: Matlab Code for Option Pricing}

\% Computes the call option price using quadl numerical integration

$\%$ Code for BPRJM Model 
$\% \mathrm{~S}_{-} \mathrm{t}=\mathrm{d}$ vector of spot stock prices

$\% \mathrm{~K}=\mathrm{d}$ vector of Strike Prices

$\%$ tau $=\mathrm{d}$ vector of time to maturity

$\% \mathrm{~h} \_\mathrm{t}=\mathrm{d}$ by 2 matrix of variance processes

function $[\mathrm{c}]=$ cPrice_QL_BPRJM_PQ$\left(S_{-}\right.$t, K,h_t,rF,tau,param $)$

$\operatorname{sigma}=\operatorname{param}(1) ; \operatorname{gamma}=\operatorname{param}(2) ;$ theta $=\operatorname{param}(3) ; \operatorname{delta}=\operatorname{param}(4)$;

rho $=\operatorname{param}(5)$;

$\mathrm{b} \_\mathrm{z}=\operatorname{param}(6) ; \mathrm{b} \_\mathrm{y}=\operatorname{param}(7) ; \mathrm{a} \_\mathrm{z}=\operatorname{param}(8) ; \mathrm{a} \_\mathrm{y}=\operatorname{param}(9)$;

h_z0 $=\operatorname{param}(10) ; \mathrm{h} \_\mathrm{y} 0=\operatorname{param}(11) ; \operatorname{chi}=\operatorname{param}(12) ; \mathrm{nu} \_3=\operatorname{param}(13)$;

$\mathrm{h} \_\mathrm{zt}=\mathrm{h} \_\mathrm{t}(:, 1)$;

$\mathrm{h} \_\mathrm{yt}=\mathrm{h} \_\mathrm{t}(:, 2)$;

$\mathrm{c}=\left(0.5^{*}\left(\mathrm{~S}_{-} \mathrm{t}^{\prime}-\mathrm{K}^{\prime} \cdot{ }^{*} \exp \left(-\mathrm{rF}{ }^{\prime} \cdot{ }^{*} \mathrm{tau}^{\prime}\right)\right)+(1 / \mathrm{pi}) * \exp \left(-\mathrm{rF}{ }^{\prime} \cdot{ }^{*} \mathrm{tau} \mathbf{u}^{\prime}\right){ }^{*} \ldots\right.$

quadl_v(@integ,0.00001,250,1e-06,[],sigma,gamma,theta,delta,rho,b_z,b_y,a_z,a_y,...

h_z0,h_y0,chi,nu_3,h_zt,h_yt,tau,S_t,K))';

function $[\mathrm{f}]=\operatorname{integ}(\mathrm{u}, \operatorname{sigma}$, gamma, theta,delta,rho,b_z,b_y,a_z,a_y,h_z0,h_y0,chi,nu_3,... h_zt,h_yt,tau,S_t,K)

$\mathrm{N}=\operatorname{numel}(\mathrm{u})$;

$\mathrm{d}=\operatorname{numel}(\mathrm{K})$;

param $=[$ sigma,gamma,theta,delta,rho,b_z,b_y,a_z,a_y,h_z0,h_y0,chi,nu_3];

$\mathrm{x}=\log \left(\mathrm{K} . / \mathrm{S}_{-} \mathrm{t}\right)^{\prime}$;

$\mathrm{h} \_\mathrm{t}=\left[\mathrm{h} \_\mathrm{zt}\right.$ h_yt $]$;

[Psi1 Psi2] = Psi_GARJV $\left(\mathrm{u}, \mathrm{h} \_\right.$t,tau,param $)$;

$\mathrm{f} 1=\operatorname{real}\left(\left(\operatorname{Psi1} .^{*} \exp \left(-1 i^{*} \mathrm{u}^{*} \mathrm{x}\right)\right) \cdot /\left(1 \mathrm{i}^{*} \operatorname{repmat}(\mathrm{u}, 1, \mathrm{~d})\right)\right)$;

$\mathrm{f} 2=\operatorname{real}\left(\left(\operatorname{Psi} 2{ }^{*} \exp \left(-1 \mathrm{i}^{*} \mathrm{u}^{*} \mathrm{x}\right)\right) \cdot /\left(1 \mathrm{i}^{*} \operatorname{repmat}(\mathrm{u}, 1, \mathrm{~d})\right)\right)$;

$\mathrm{f}=\operatorname{repmat}\left(\mathrm{S}_{-} \mathrm{t}^{\prime}, \mathrm{N}, 1\right){ }^{*} \mathrm{f} 1-\operatorname{repmat}\left(\mathrm{K}^{\prime}, \mathrm{N}, 1\right){ }^{*} \mathrm{f} 2$;

$\% \mathrm{u}$ is a $\mathrm{N}$-column vector

$\%$ tau is a d-column vector of maturities

$\%$ Output is $\mathrm{N}$ by d matrix

function $\left[\right.$ Psi1 Psi2] = Psi_GARJV $\left(\mathrm{u}_{\text {, }} \mathrm{h}_{-} \mathrm{t}, \mathrm{tau}, \mathrm{param}\right)$

$\mathrm{u} 1=1+1 \mathrm{i}^{*} \mathrm{u}$;

$\mathrm{u} 2=1 \mathrm{i}^{*} \mathrm{u}$;

$\mathrm{h} \_\mathrm{zt}=\mathrm{h} \_\mathrm{t}(:, 1)$;

$\mathrm{h} \_\mathrm{yt}=\mathrm{h} \_\mathrm{t}(:, 2)$;

$\mathrm{N}=\operatorname{numel}(\mathrm{u})$;

$\mathrm{T}=\max (\mathrm{tau})$;

C1Mat1 $=\operatorname{zeros}(\mathrm{N}, \mathrm{T})$; 
$\mathrm{C} 2 \mathrm{Mat} 1=\operatorname{zeros}(\mathrm{N}, \mathrm{T})$

DMat1 $=\operatorname{zeros}(\mathrm{N}, \mathrm{T})$

C1Mat2 $=\operatorname{zeros}(\mathrm{N}, \mathrm{T})$;

$\mathrm{C} 2 \mathrm{Mat} 2=\operatorname{zeros}(\mathrm{N}, \mathrm{T})$;

DMat2 $=\operatorname{zeros}(\mathrm{N}, \mathrm{T})$

$\% \mathrm{C}$ and $\mathrm{D}$ for maturity 1

[C1Mat1(:,1) C2Mat1(:,1) DMat1(:,1)] = A12B(u1,zeros(N,1),zeros(N,1),param);

[C1Mat2(:,1) C2Mat2(:,1) DMat2(:,1)] = A12B(u2,zeros(N,1),zeros(N,1),param);

$\%$ Recursion up to $\mathrm{M}$

for $\mathrm{t}=2: \mathrm{T}$

[C1Mat1(:,t) C2Mat1(:,t) DMat1(:,t)] = A12B(u1,C1Mat1(:,t-1),C2Mat1(:,t-1),param);

[C1Mat2(:,t) C2Mat2(:,t) DMat2(:,t)] = A12B(u2,C1Mat2(:,t-1),C2Mat2(:,t-1),param);

$\operatorname{DMat1}(:, \mathrm{t})=\operatorname{DMat} 1(:, \mathrm{t})+\operatorname{DMat1}(:, \mathrm{t}-1)$;

$\operatorname{DMat} 2(:, \mathrm{t})=\operatorname{DMat} 2(:, \mathrm{t})+\operatorname{DMat} 2(:, \mathrm{t}-1)$;

end

Psi1 $=\exp ($ C1Mat1(:,tau).*repmat(h_zt',N,1) + C2Mat1(:,tau).*repmat(h_yt',N,1) + DMat1(:,tau));

Psi2 $=\exp \left(\mathrm{C} 1 \mathrm{Mat} 2(:, \mathrm{tau}) .{ }^{*}\right.$ repmat(h_zt',N,1) + C2Mat2(:,tau).*repmat(h_yt',N,1) + DMat2(:,tau));

$\% \mathrm{u}, \mathrm{w} \_\mathrm{R}, \mathrm{w} \_\mathrm{RV}$ are N-column vectors

function $[\mathrm{A} 1 \mathrm{~A} 2 \mathrm{~B}]=\mathrm{A} 12 \mathrm{~B}\left(\mathrm{u}, \mathrm{v} \_\mathrm{z}, \mathrm{v} \_\mathrm{y}, \mathrm{param}\right)$

$\%$ Set $\mathrm{rF}=0.05 / 365$ for the ease of computation

$\mathrm{rF}=0.05 / 365$

sigma $=\operatorname{param}(1) ; \operatorname{gamma}=\operatorname{param}(2) ;$ theta $=\operatorname{param}(3) ;$ delta $=\operatorname{param}(4) ;$

rho $=\operatorname{param}(5)$;

b_z $=\operatorname{param}(6) ; \mathrm{b} \_\mathrm{y}=\operatorname{param}(7) ; \mathrm{a} \_\mathrm{z}=\operatorname{param}(8) ; \mathrm{a} \_\mathrm{y}=\operatorname{param}(9)$;

h_z0 $=\operatorname{param}(10) ; \mathrm{h} \_y 0=\operatorname{param}(11) ; \operatorname{chi}=\operatorname{param}(12) ; \mathrm{nu} \_3=\operatorname{param}(13)$;

gamma_s = gamma - chi;

theta_s $=$ theta + delta^ $2^{*}$ nu_3;

$\mathrm{xi} \_\mathrm{s}=\exp ($ theta_s+delta^2/2) - 1 ;

omega_z $=\mathrm{h} \_z 0^{*}\left(1-\left(\mathrm{b} \_\mathrm{z}+\mathrm{a} \_\mathrm{z}\right)\right)$;

omega_y $=\mathrm{h} \_\mathrm{y} 0 *\left(1-\left(\mathrm{b} \_\mathrm{y}+\mathrm{a} \_\mathrm{y}^{*}\left(\operatorname{theta}^{\wedge} 2+\operatorname{delta}^{\wedge} 2\right)\right)\right)$;

$\mathrm{v} 1=\mathrm{v} \_\mathrm{z}^{*} \mathrm{a} \_\mathrm{z} ;$

$\mathrm{v} 2=\mathrm{v} \_\mathrm{y} * \mathrm{a} \_\mathrm{y}$

$\mathrm{v} 3=-0.5^{*} \log \left(1-2^{*} \mathrm{v} 2 * \operatorname{delta}^{\wedge} 2\right)+\mathrm{u}^{*}$ theta_s $+\mathrm{v} 2 *$ theta_s^ $2+\ldots$

$\left(\left(\mathrm{u}^{*}\right.\right.$ delta $+2{ }^{*}$ theta_s* ${ }^{*} 2 *$ delta $\left.) . \wedge 2\right) . /(2 *(1-2 * \mathrm{v} 2 *$ delta^ 2$))$;

$\mathrm{A} 1=-0.5^{*} \mathrm{u}+\mathrm{v} \_\mathrm{z}^{*} \mathrm{~b} \_\mathrm{z}+\mathrm{v} 1+\left(\text { gamma_s } \mathrm{s}^{\wedge} 2 \text {-gamma^} 2\right)^{*} \mathrm{v} 1+0.5^{*}(1-\text { rho^2 })^{*} \mathrm{u} \cdot \cdot^{\wedge} 2+$ $\left(\left(\mathrm{u}^{*}\right.\right.$ rho- $2^{*}$ sigma* ${ }^{*}{ }^{*}$ gamma_s $\left.) \cdot \wedge 2\right) \cdot /\left(2 *\left(1-2 *\right.\right.$ sigma* $\left.\left.{ }^{*} 1\right)\right)$; 


$$
\begin{aligned}
& \text { A2 }=-\mathrm{xi} \_\mathrm{s}^{*} \exp \left(\text { theta }^{*} \mathrm{nu} \_3+0.5^{*} \operatorname{delta}^{\wedge} 2^{*} \mathrm{nu} \_3^{\wedge} 2\right)^{*} \mathrm{u}+\mathrm{v} \_\mathrm{y}^{*} \mathrm{~b} \_\mathrm{y}+\ldots \\
& (\exp (\mathrm{v} 3)-1)^{*} \exp \left(\operatorname{theta}^{*} \mathrm{nu} \_3+0.5^{*} \operatorname{delta}^{\wedge} 2^{*} \mathrm{nu} \_3^{\wedge} 2\right) \\
& \mathrm{B}=\mathrm{u}^{*} \mathrm{rF}+\mathrm{v} \_\mathrm{z}^{*} \text { omega_z}+\mathrm{v} \_\mathrm{y}^{*} \text { omega_y }-\mathrm{v} 1^{*} \text { sigma }-0.5^{*} \log \left(1-2^{*} \text { sigma }^{*} \mathrm{v} 1\right)
\end{aligned}
$$


Figure 1: Daily Returns and Realized Variation Measures.
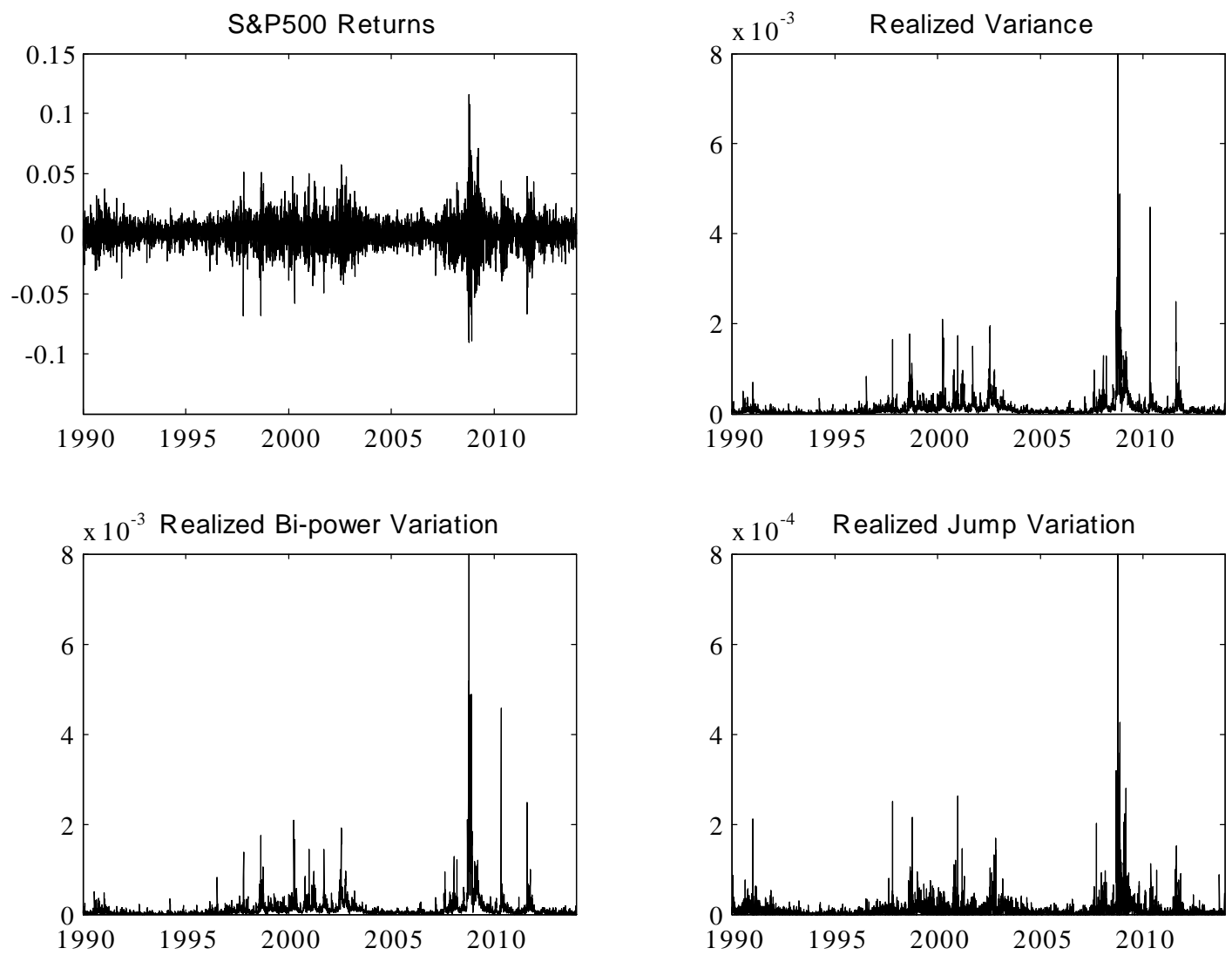

Notes: The top left panel shows the daily log returns on the S\&P500 index. The top right panel shows the daily realized variance computed from averages of the sum of squared overlapping 5-minute returns. The bottom left panel shows the realized bipower variation computed using the method in Barndorff-Nielsen and Shephard (2004). The bottom right panel shows the realized jump variation constructed as the difference between realized variance and realized bipower variation. The sample starts 2 January 1990 and ends 31 December 2013. 
Figure 2: Autocorrelations of Daily Returns and Realized Variation Measures.
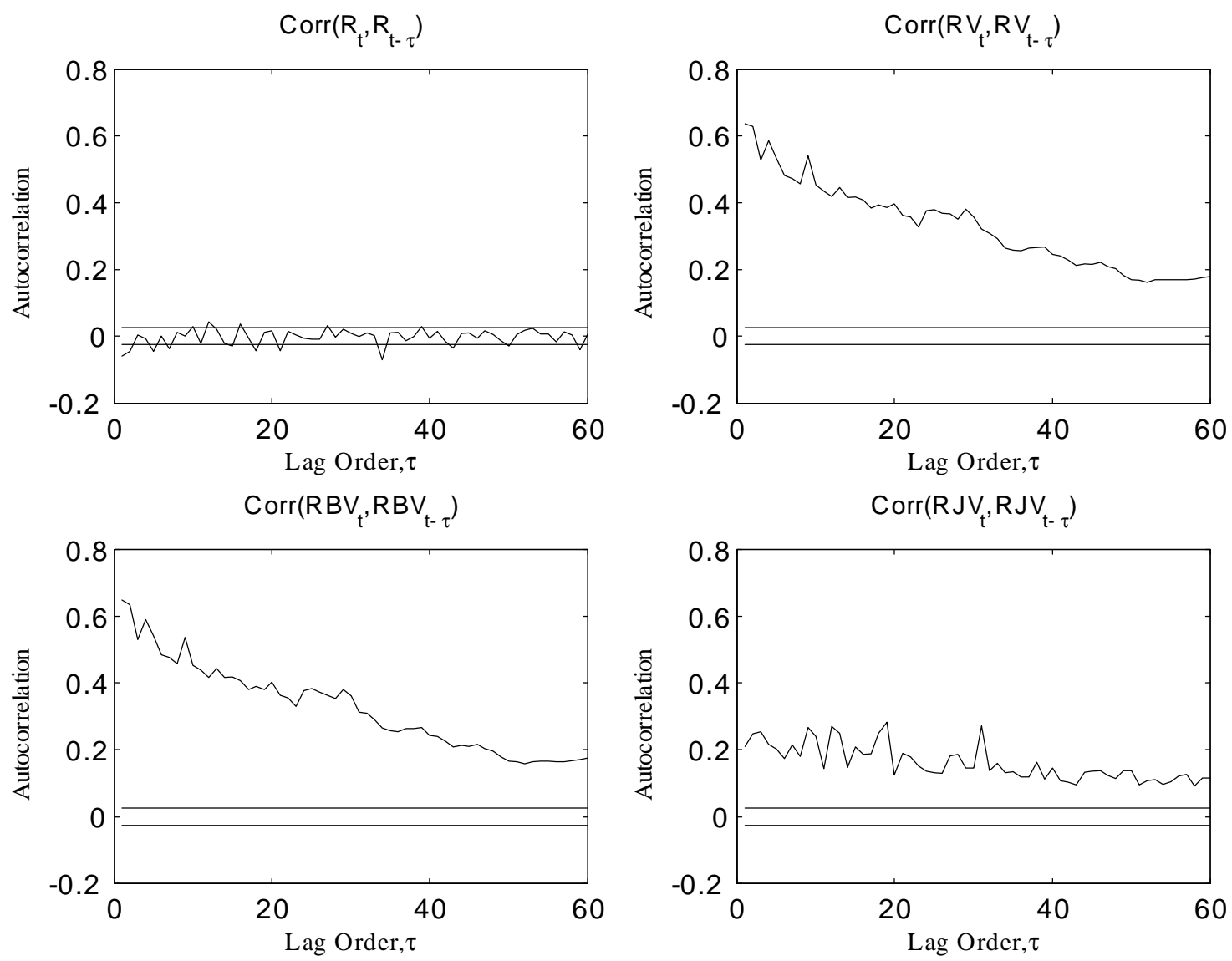

Notes: We report the sample autocorrelation functions for lag 1 through 60 trading days for returns (top left panel), realized volatility (top right panel), realized bipower variation (bottom left panel), and realized jump variation (bottom right panel). The sample starts 2 January 1990 and ends 31 December 2013. 
Figure 3: Daily Conditional Volatility.
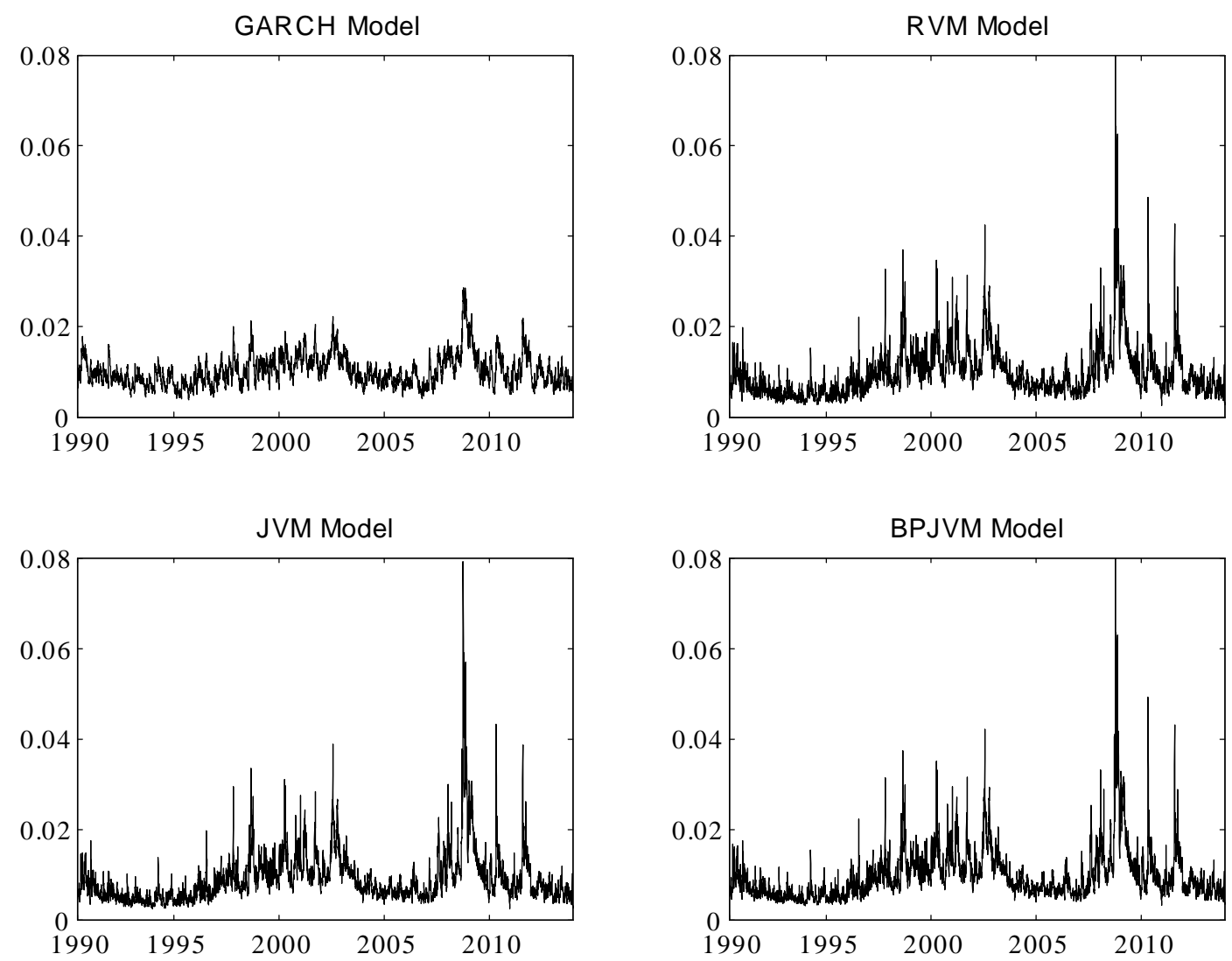

Notes: We plot the daily model-based conditional volatility for the four models we consider: the benchmark Heston-Nandi GARCH model (top left), the RVM model based on realized volatility (top right), the RJM model based on realized jump variation only (bottom left), and the full BPRJM model that separately uses bipower variation and realized jump variation (bottom right). We use the parameter estimates in Table 1 . The sample starts 2 January 1990 and ends 31 December 2013. 
Figure 4: Conditional Volatility of Variance.
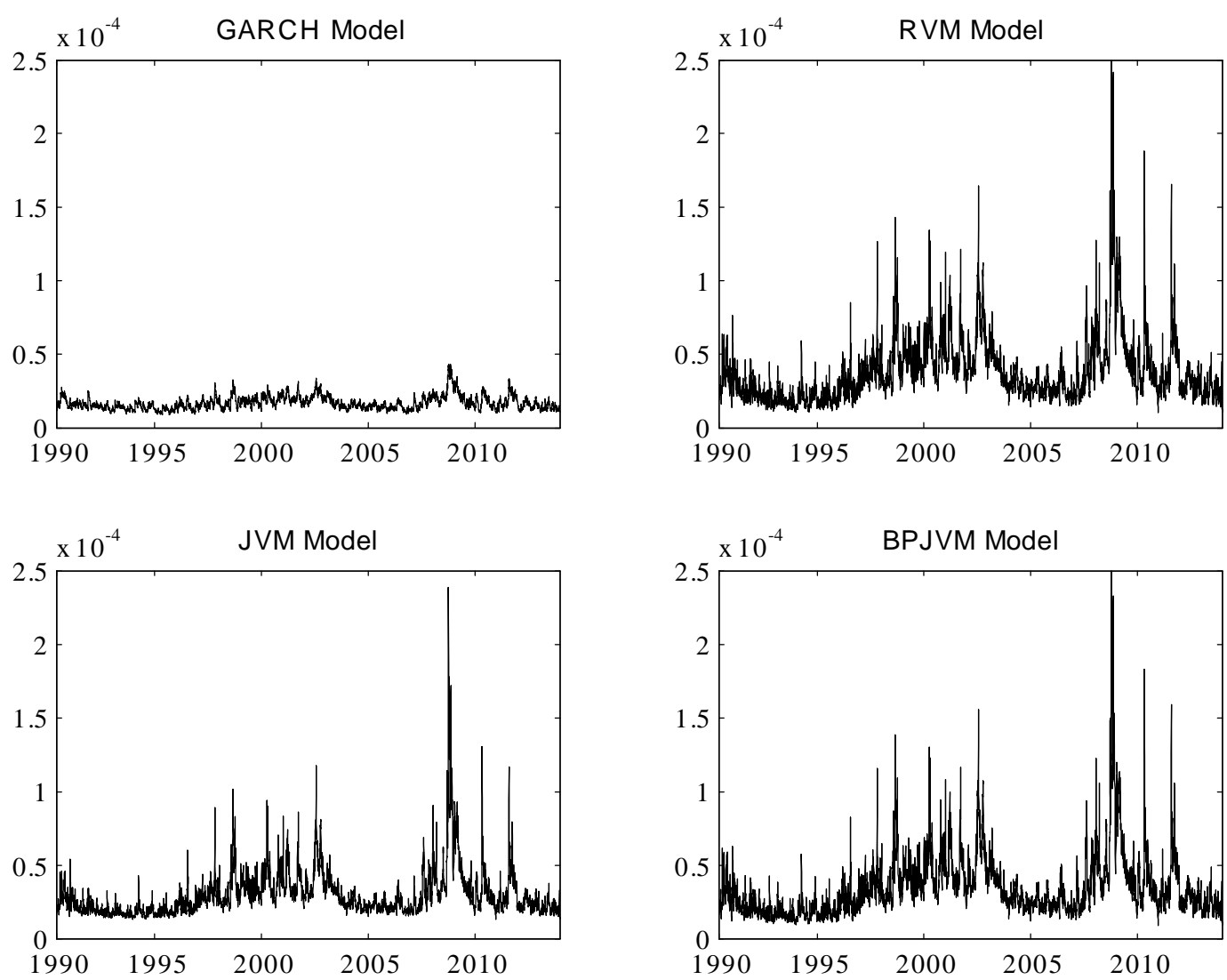

Notes: We plot the daily model-based conditional volatility of variance for the four models we consider: the benchmark Heston-Nandi GARCH model (top left), the RVM model based on realized volatility (top right), the RJM model based on realized jump variation only (bottom left), and the full BPRJM model that separately uses realized bipower variation and realized jump variation (bottom right). We use the parameter estimates in Table 1. The sample starts 2 January 1990 and ends 31 December 2013. 
Figure 5: Daily Correlation of Returns and Variance.
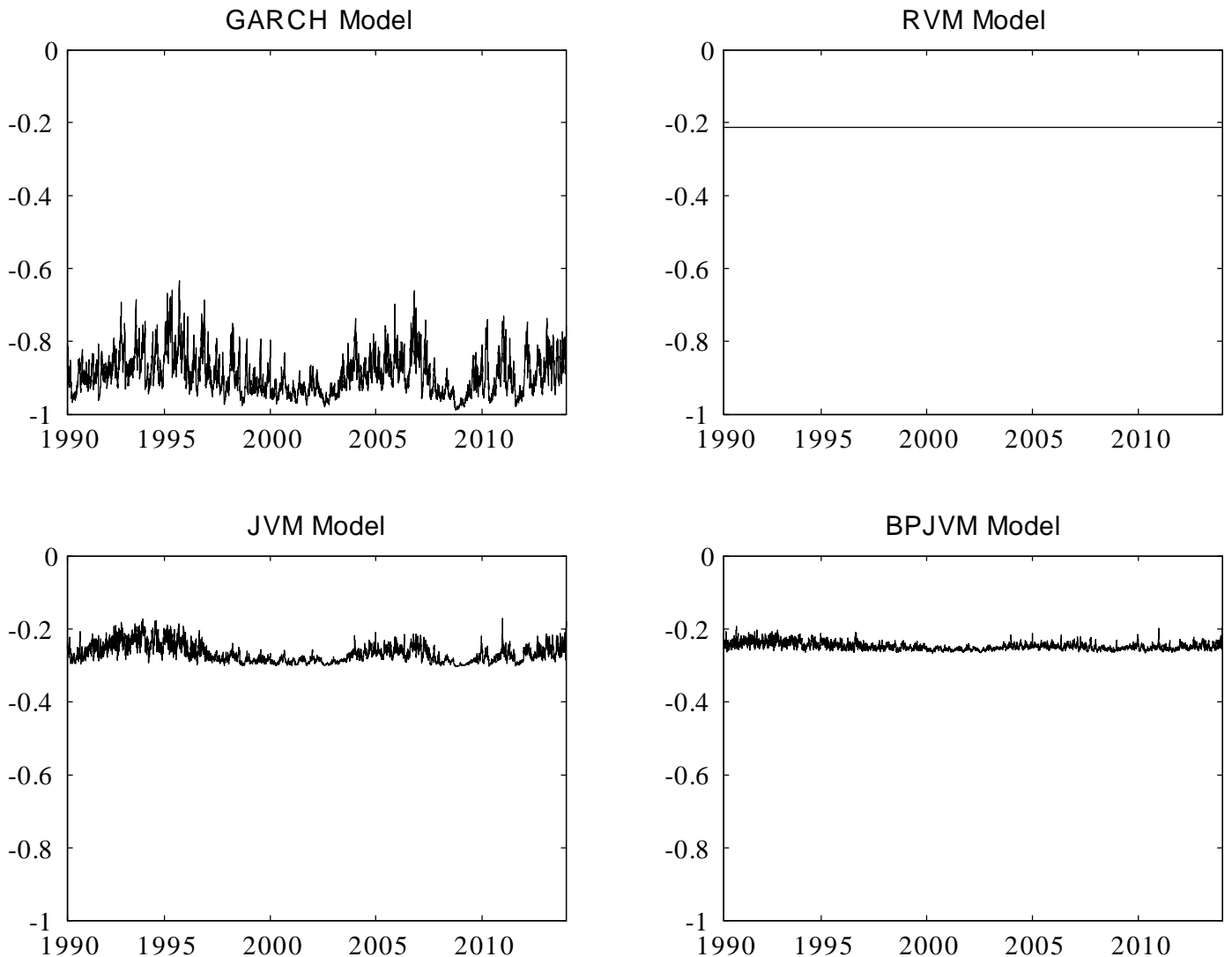

Notes: We plot the daily model-based conditional correlation of returns and variance for the four models we consider: the benchmark Heston-Nandi GARCH model (top left), the RVM model based on realized volatility (top right), the RJM model based on realized jump variation only (bottom left), and the full BPRJM model that separately uses realized bipower variation and realized jump variation (bottom right). We use the parameter estimates in Table 1. The sample starts 2 January 1990 and ends 31 December 2013. 
Figure 6: Realized Volatility and Predicted Volatility from Models.
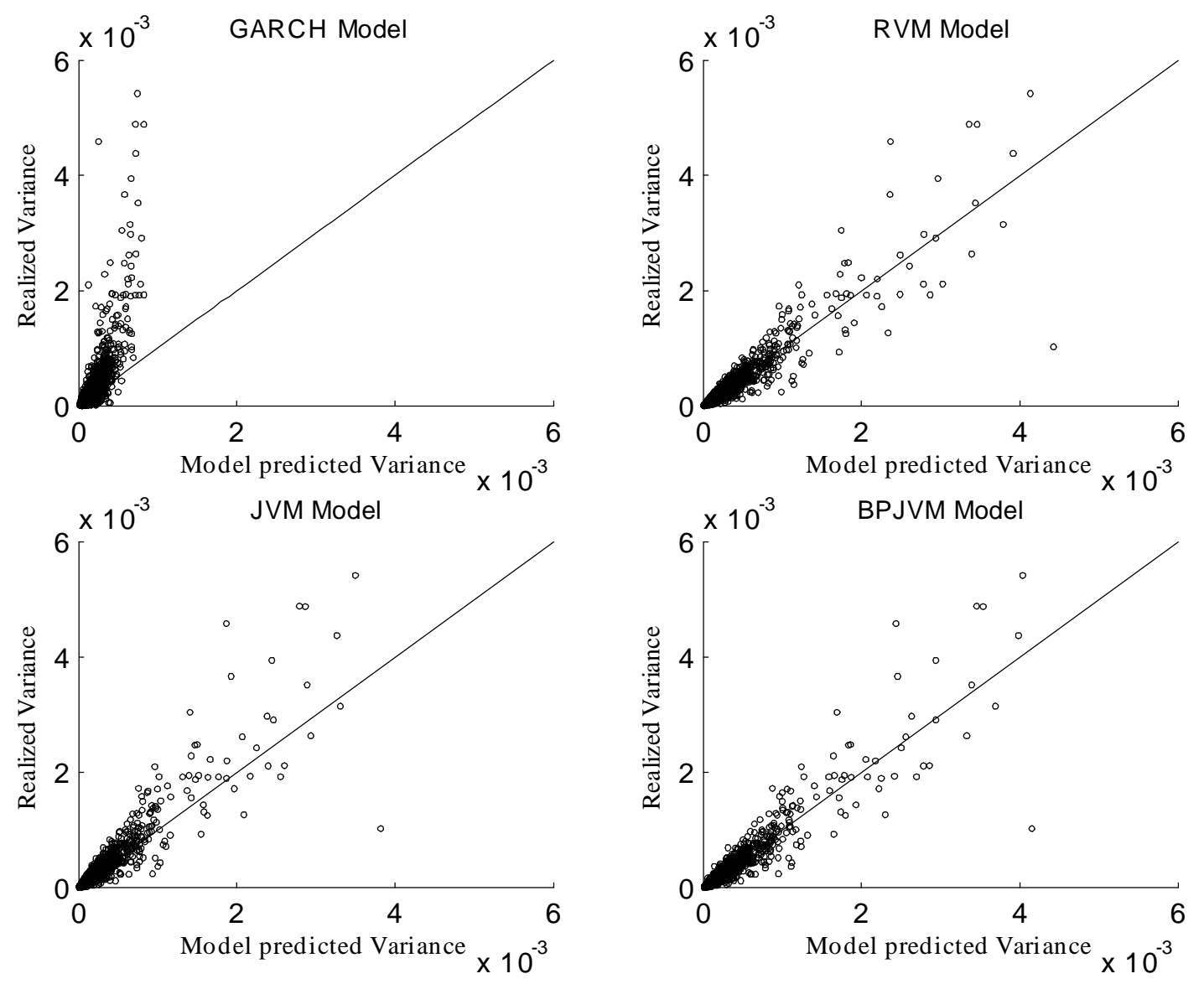

Notes: We scatter plot the ex-post realized variance (vertical axis) against the modelpredicted total variance (horizontal axis) for each of our models: the benchmark HestonNandi GARCH model (top left), the RVM model based on realized volatility (top right), the RJM model based on realized jump variation only (bottom left), and the full BPRJM model that separately uses realized bipower variation and realized jump variation (bottom right). We use the parameter estimates in Table 1. The sample starts 2 January 1990 and ends 31 December 2013. 
Figure 7: Weekly Implied Root Mean Squared Error from At-the-Money Options.
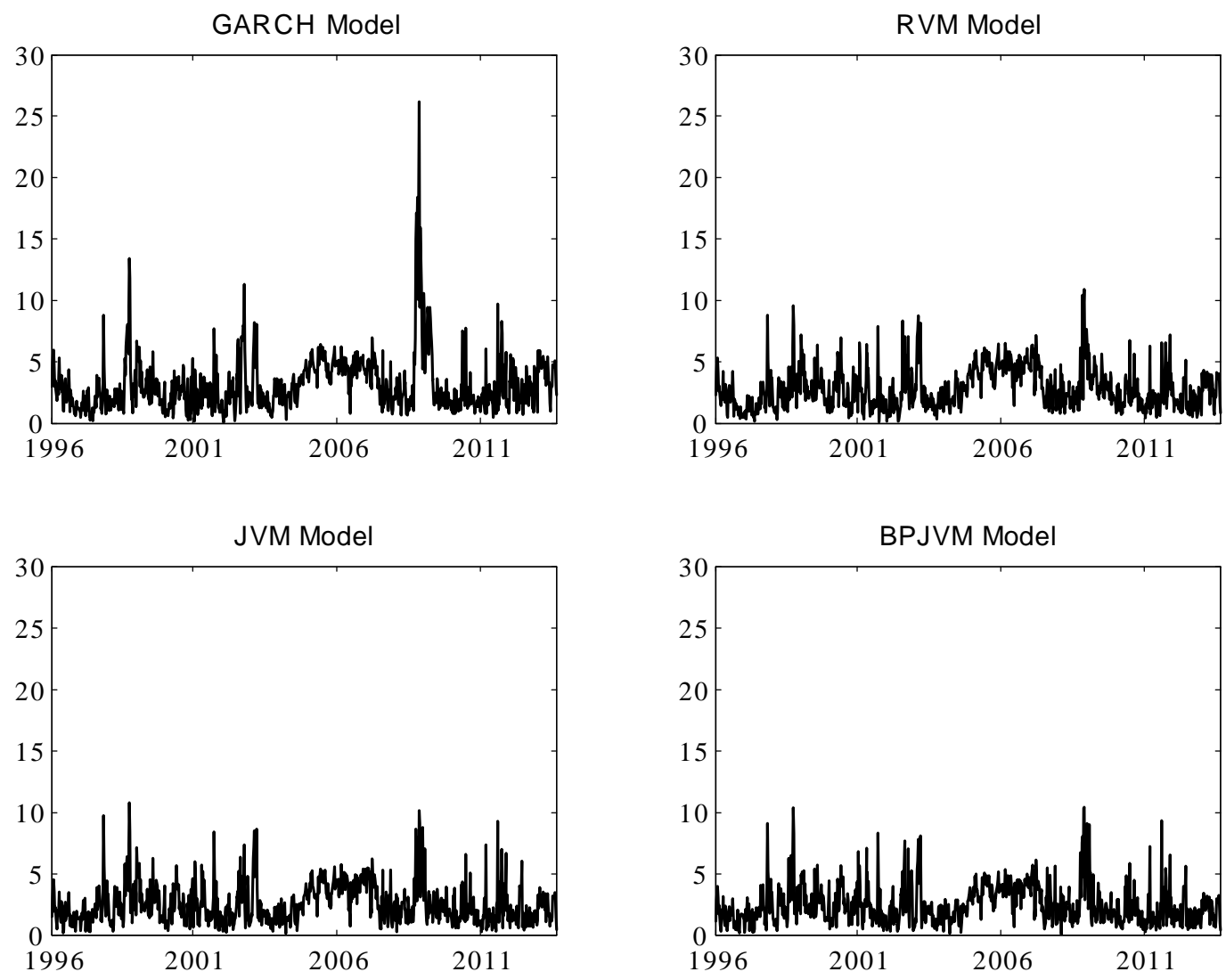

Notes: We plot the weekly implied volatility root mean squared error for at-the-money options for each of our models: the benchmark Heston-Nandi GARCH model (top left), the RVM model based on realized volatility (top right), the RJM model based on realized jump variation only (bottom left), and the full BPRJM model that separately uses realized bipower variation and realized jump variation (bottom right). We use the parameter estimates in Table 3. The option sample starts 2 January 1996 and ends 28 August 2013. 
Figure 8: Model-Based, Risk-Neutral Higher Moments. 6-Month Horizon.
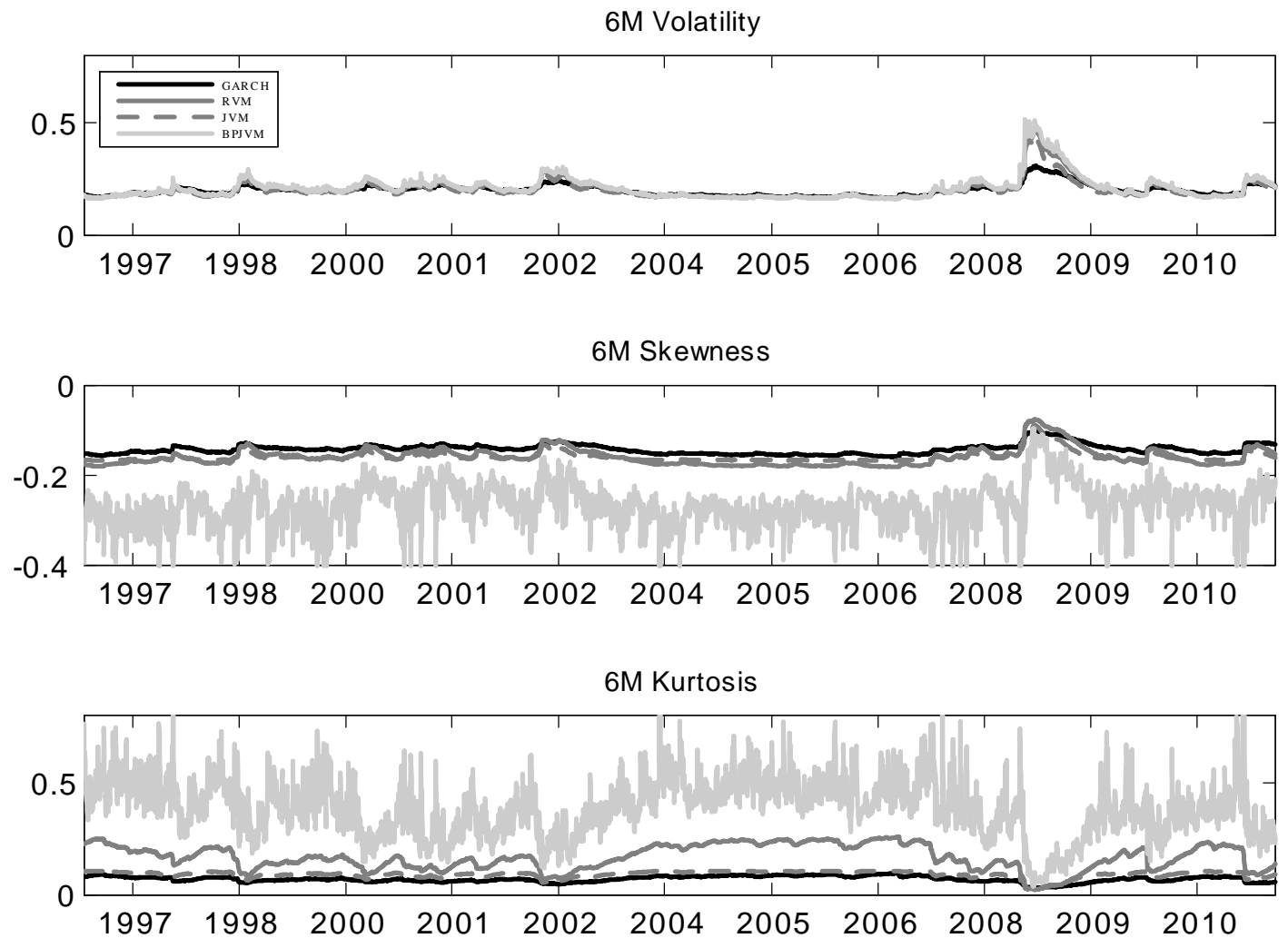

Notes: We plot the 6-month risk-neutral volatility, skewness and kurtosis implied by each of our models: the benchmark Heston-Nandi GARCH model (solid black), the RVM model based on realized volatility (solid dark grey), the RJM model based on realized jump variation only (dashed dark grey), and the full BPRJM model that separately uses realized bipower variation and realized jump variation (solid light grey). We use the parameter estimates in Table 3. The option sample starts 2 January 1996 and ends 28 August 2013. 
Figure 9: Autocorrelations of Weekly Vega-Weighted Root Mean Squared Errors from ATM Options
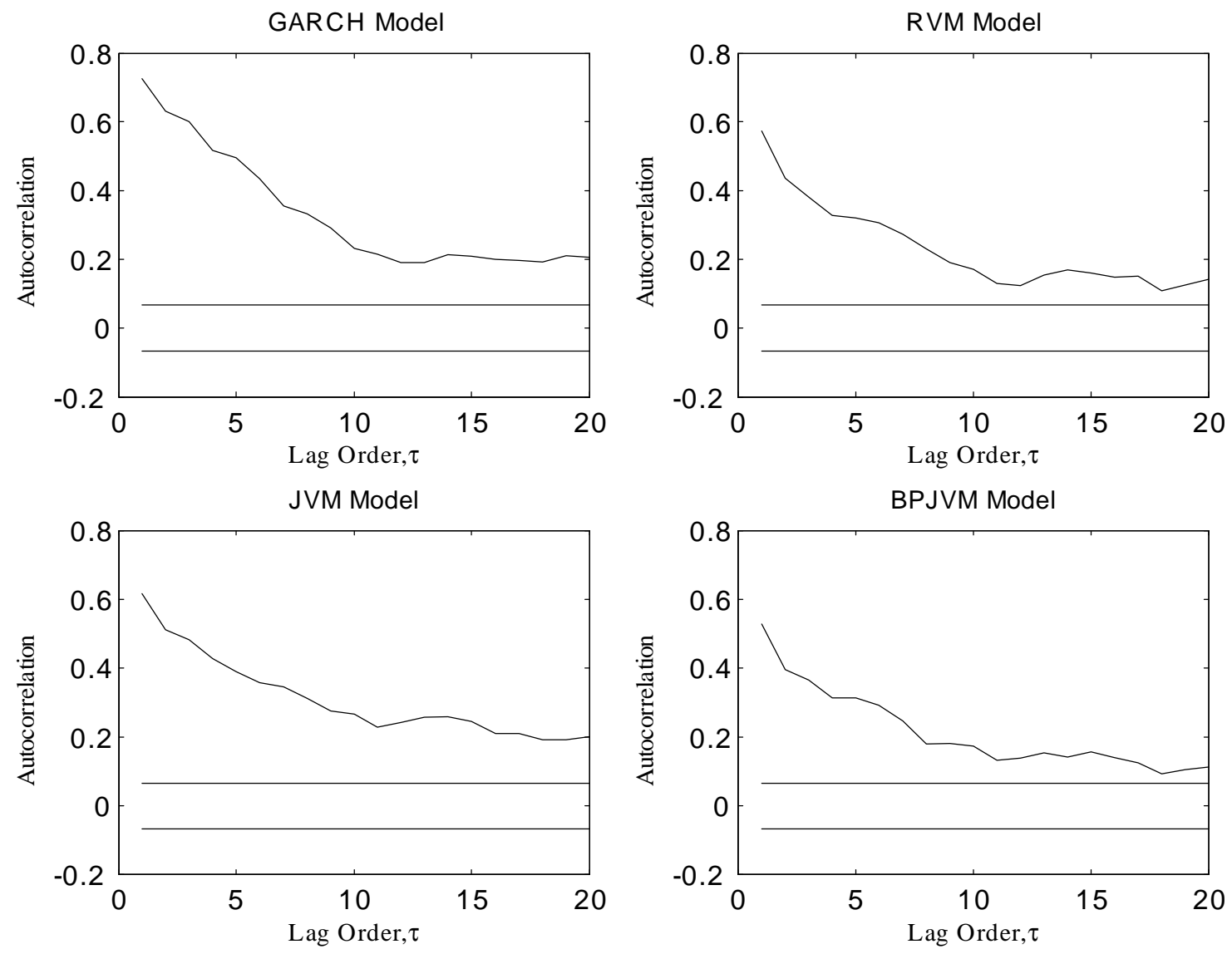

Notes: We plot autocorrelations of the weekly vega-weighted root mean squared error for at-the-money options from each of our models: the benchmark Heston-Nandi GARCH model (top left), the RVM model based on realized volatility (top right), the RJM model based on realized jump variation only (bottom left), and the full BPRJM model that separately uses realized bipower variation and realized jump variation (bottom right). We use the parameter estimates in Table 3. The option sample starts 2 January 1996 and ends 28 August 2013. 
Table 1: Maximum Likelihood Estimation on Daily S\&P500 Returns and Realized Measures. 1990-2013

\begin{tabular}{|c|c|c|c|c|c|c|c|c|}
\hline \multirow{3}{*}{$\begin{array}{c}\text { Parameters } \\
\lambda_{z}\end{array}$} & \multicolumn{2}{|c|}{ GARCH } & \multicolumn{2}{|c|}{ RVM } & \multicolumn{2}{|c|}{$\underline{\text { JVM }}$} & \multicolumn{2}{|c|}{ BPJVM } \\
\hline & Estimate & $\underline{\text { Std Error }}$ & $\underline{\text { Estimate }}$ & $\underline{\text { Std Error }}$ & $\underline{\text { Estimate }}$ & $\underline{\text { Std Error }}$ & Estimate & $\underline{\text { Std Error }}$ \\
\hline & $\overline{4.30 \mathrm{E}-01}$ & $\overline{(6.87 \mathrm{E}-01)}$ & $\overline{4.40 \mathrm{E}-01}$ & $(1.12 \mathrm{E}+00)$ & & & $4.19 \mathrm{E}-01$ & $(1.86 \mathrm{E}+00)$ \\
\hline$\lambda_{y}$ & & & & & $2.06 \mathrm{E}-06$ & $(2.31 \mathrm{E}-05)$ & $9.13 \mathrm{E}-05$ & $(4.67 \mathrm{E}-05)$ \\
\hline$\alpha$ & 4.87E-06 & $(1.57 \mathrm{E}-07)$ & & & & & & \\
\hline$\beta$ & $8.50 \mathrm{E}-01$ & $(1.13 \mathrm{E}-02)$ & & & & & & \\
\hline$\gamma$ & $1.53 \mathrm{E}+02$ & $(7.66 \mathrm{E}+00)$ & $7.40 \mathrm{E}+03$ & $(1.81 \mathrm{E}+01)$ & & & $1.45 \mathrm{E}+04$ & $(6.23 \mathrm{E}+01)$ \\
\hline$\omega_{z}$ & $4.73 \mathrm{E}-14$ & & $2.35 \mathrm{E}-08$ & & & & $7.06 \mathrm{E}-08$ & \\
\hline$\omega_{y}$ & & & & & $3.38 \mathrm{E}-02$ & & $8.27 \mathrm{E}-02$ & \\
\hline$\sigma$ & & & $5.28 \mathrm{E}-07$ & $(2.12 \mathrm{E}-07)$ & & & $2.51 \mathrm{E}-07$ & $(1.71 \mathrm{E}-08)$ \\
\hline$\theta$ & & & & & $-7.98 \mathrm{E}-04$ & $(3.08 \mathrm{E}-05)$ & $1.42 \mathrm{E}-05$ & $(1.98 \mathrm{E}-05)$ \\
\hline$\delta$ & & & & & $4.40 \mathrm{E}-03$ & $(4.13 \mathrm{E}-06)$ & $1.62 \mathrm{E}-03$ & $(3.20 \mathrm{E}-06)$ \\
\hline$\rho$ & & & $2.14 \mathrm{E}-01$ & $(7.38 \mathrm{E}-02)$ & & & 2.67E-01 & $(9.52 \mathrm{E}-02)$ \\
\hline$b_{z}$ & & & $5.05 \mathrm{E}-01$ & $(3.64 \mathrm{E}-02)$ & & & 4.87E-01 & $(4.21 \mathrm{E}-02)$ \\
\hline$b_{y}$ & & & & & $5.46 \mathrm{E}-01$ & $(3.60 \mathrm{E}-02)$ & $9.16 \mathrm{E}-01$ & $(2.14 \mathrm{E}-02)$ \\
\hline$a_{z}$ & & & $4.95 \mathrm{E}-01$ & $(3.54 \mathrm{E}-02)$ & & & $5.12 \mathrm{E}-01$ & $(4.36 \mathrm{E}-02)$ \\
\hline$a_{y}$ & & & & & $1.94 \mathrm{E}+04$ & $(1.21 \mathrm{E}+02)$ & $2.41 \mathrm{E}+04$ & $(6.31 \mathrm{E}+02)$ \\
\hline$E\left[h_{z, t}\right]$ & $1.16 \mathrm{E}-04$ & & $1.35 \mathrm{E}-04$ & & & & $1.24 \mathrm{E}-04$ & \\
\hline $\mathrm{E}\left[h_{y, t}\right]$ & & & & & $5.70 \mathrm{E}+00$ & & $4.04 \mathrm{E}+00$ & \\
\hline roperties & & & & & & & & \\
\hline $\begin{array}{l}\text { Tolatility } \\
\text { Persistence }\end{array}$ & 18.34 & & 18.34 & & 18.34 & & 18.34 & \\
\hline eturns & 0.9635 & & & & & & & \\
\hline $\mathrm{V}$ & & & 0.9998 & & 0.9340 & & & \\
\hline$B V$ & & & & & & & 0.9998 & \\
\hline JV & & & & & & & 0.9795 & \\
\hline hoods & & & & & & & & \\
\hline,$\overline{R B V}$, and $\mathrm{RJV}$ & & & & & & & 129,226 & \\
\hline zed on Returns and RV & & & 68,212 & & 68,783 & & 69,656 & \\
\hline zed on Returns & 19,312 & & 19,515 & & 19,515 & & 19,522 & \\
\hline
\end{tabular}

Model Properties

Average Volatility

Volatility Persistence

From Returns

From RV

From RBV

From RJV

Log-Likelihoods

Returns, RBV, and RJV

Maximized on Returns and RV

Maximized on Returns
19,312
19,515
19,515
19,522

Notes: Using daily returns and daily realized variation measures, we estimate our four models using maximum likelihood criteria. For comparison, the last row reports likelihood values when all models are estimated on returns only. The second-to-last row reports likelihood values when the RVM, JVM, and BPJVM models are estimated on returns and realized variance. The third-to-last row reports the likelihood value when the BPJVM model is estimated on returns, bipower variation and jump variation. The parameter values reported correspond to the second-last row for RVM and JVM and to the third-last row for the BPJVM model. The sample is from 2 January 1990 through 31 December 2013. Standard errors are reported in parentheses. Variance targeting is used to fix the $\omega$ parameters. 
Table 2: S\&P500 Index Option Data by Moneyness, Maturity and VIX Level. 1996-2013

\begin{tabular}{|c|c|c|c|c|c|c|c|}
\hline By Moneyness & $\underline{\text { Delta }<0.3}$ & $\underline{0.3<\text { Delta }<0.4}$ & $\underline{0.4<\text { Delta }<0.5}$ & $\underline{0.5<\text { Delta }<0.6}$ & $\underline{0.6<\text { Delta }<0.7}$ & $\underline{\text { Delta }}>0.7$ & $\underline{\text { All }}$ \\
\hline Number of Contracts & 3,788 & 1,391 & 1,781 & 2,846 & 2,746 & 8,731 & $21 \overline{283}$ \\
\hline Average Price & 7.85 & 20.94 & 32.28 & 45.30 & 65.93 & 132.41 & 74.35 \\
\hline Average Implied Volatility & 16.72 & 18.40 & 19.31 & 20.40 & 21.71 & 25.09 & 21.62 \\
\hline Average Bid-Ask Spread & 1.046 & 1.674 & 1.955 & 2.018 & 1.834 & 1.228 & 1.470 \\
\hline By Maturity & $\underline{\mathrm{DTM}}<30$ & $\underline{30<\mathrm{DTM}<60}$ & $\underline{60<\mathrm{DTM}<90}$ & $\underline{90<\mathrm{DTM}<120}$ & $\underline{120<\mathrm{DTM}<150}$ & $\underline{\mathrm{DTM}}>150$ & $\underline{\text { All }}$ \\
\hline Number of Contracts & 2,725 & 6,480 & 5,053 & 2,869 & $1,1,974$ & 2,182 & $21 \overline{283}$ \\
\hline Average Price & 41.26 & 61.01 & 76.44 & 92.30 & 97.88 & 105.59 & 74.35 \\
\hline Average Implied Volatility & 20.21 & 21.28 & 21.73 & 22.94 & 22.08 & 21.95 & 21.62 \\
\hline Average Bid-Ask Spread & 0.820 & 1.231 & 1.579 & 1.872 & 1.800 & 1.910 & 1.470 \\
\hline By VIX Level & $\underline{\mathrm{VIX}}<15$ & $\underline{15}<$ VIX $<20$ & $20<$ VIX $<25$ & $25<\mathrm{VIX}<30$ & $\underline{30<\mathrm{VIX}<35}$ & VIX $>35$ & All \\
\hline Number of Contracts & 3,962 & 6,133 & 5,996 & 2,456 & 1,240 & 1,496 & 21,283 \\
\hline Average Price & 57.95 & 66.90 & 80.75 & 85.77 & 85.33 & 94.86 & 74.35 \\
\hline Average Implied Volatility & 13.61 & 18.04 & 22.45 & 26.24 & 30.22 & 39.42 & 21.62 \\
\hline Average Bid-Ask Spread & 1.055 & 1.301 & 1.446 & 1.704 & 1.811 & 2.683 & 1.470 \\
\hline
\end{tabular}

Notes: We use 21,283 S\&P500 index option contracts from OptionMetrics. The contracts have been subjected to standard filters as described in the text. The top panel reports the contracts sorted by moneyness defined using the Black-Scholes delta. The second panel reports the contracts sorted by days to maturity (DTM). The third panel reports the contract sorted by the VIX level on the day corresponding to the option quote. 
Table 3: Maximum Likelihood Estimation on Daily S\&P500 Returns, Realized Measures and Options. 1996-2013

\begin{tabular}{|c|c|c|c|c|c|c|c|c|}
\hline \multirow[b]{2}{*}{$\begin{array}{c}\text { Parameters } \\
\lambda_{z}\end{array}$} & \multicolumn{2}{|c|}{ GARCH } & \multicolumn{2}{|c|}{$\underline{\text { RVM }}$} & \multicolumn{2}{|c|}{ JVM } & \multicolumn{2}{|c|}{ BPJVM } \\
\hline & $\frac{\text { Estimate }}{1.40 \mathrm{E}+01}$ & $\frac{\text { Std Error }}{(1.03 \mathrm{E}+01)}$ & $\frac{\text { Estimate }}{9.17 \mathrm{E}-01}$ & $\frac{\text { Std Error }}{(7.91 \mathrm{E}-01)}$ & Estimate & $\underline{\text { Std Error }}$ & $\frac{\text { Estimate }}{1.36 \mathrm{E}+00}$ & $\frac{\text { Std Error }}{(9.69 \mathrm{E}-01)}$ \\
\hline$\lambda_{y}$ & & & & & $1.45 \mathrm{E}-10$ & $(4.20 \mathrm{E}-05)$ & $2.18 \mathrm{E}-05$ & $(1.70 \mathrm{E}-05)$ \\
\hline$\alpha$ & $9.01 \mathrm{E}-07$ & $(1.86 \mathrm{E}-08)$ & & & & & & \\
\hline$\beta$ & $9.88 \mathrm{E}-01$ & (6.09E-04) & & & & & & \\
\hline$\gamma$ & $6.22 \mathrm{E}+01$ & $(5.51 \mathrm{E}+00)$ & $3.04 \mathrm{E}+01$ & (2.03E-01) & & & $3.72 \mathrm{E}+01$ & $(2.68 \mathrm{E}-01)$ \\
\hline$\omega_{z}$ & $1.64 \mathrm{E}-08$ & & $7.68 \mathrm{E}-07$ & & & & $6.12 \mathrm{E}-07$ & \\
\hline$\omega_{y}$ & & & & & $1.64 \mathrm{E}-06$ & & $7.49 \mathrm{E}-03$ & \\
\hline$\sigma$ & & & $1.41 \mathrm{E}-04$ & $(2.69 \mathrm{E}-07)$ & & & $1.34 \mathrm{E}-04$ & $(2.56 \mathrm{E}-07)$ \\
\hline$\theta$ & & & & & $-2.00 \mathrm{E}-03$ & (3.39E-05) & $-1.25 \mathrm{E}-03$ & (1.35E-05) \\
\hline$\delta$ & & & & & $5.61 \mathrm{E}-03$ & (7.49E-06) & $1.38 \mathrm{E}-03$ & $(5.63 \mathrm{E}-06)$ \\
\hline$\rho$ & & & $9.14 \mathrm{E}-01$ & $(2.41 \mathrm{E}-03)$ & & & $8.79 \mathrm{E}-01$ & $(2.48 \mathrm{E}-03)$ \\
\hline$b_{z}$ & & & $9.73 \mathrm{E}-01$ & $(2.05 \mathrm{E}-04)$ & & & $9.76 \mathrm{E}-01$ & $(1.92 \mathrm{E}-04)$ \\
\hline$b_{y}$ & & & & & $9.61 \mathrm{E}-01$ & $(3.02 \mathrm{E}-04)$ & $6.63 \mathrm{E}-01$ & $(2.96 \mathrm{E}-03)$ \\
\hline$a_{z}$ & & & $1.48 \mathrm{E}-02$ & $(9.86 \mathrm{E}-05)$ & & & $1.32 \mathrm{E}-02$ & $(9.05 \mathrm{E}-05)$ \\
\hline$a_{y}$ & & & & & $7.07 \mathrm{E}+02$ & $(8.59 \mathrm{E}+00)$ & $9.55 \mathrm{E}+04$ & $(8.87 \mathrm{E}+02)$ \\
\hline$E\left[h_{z, t}\right]$ & $1.03 \mathrm{E}-04$ & $(1.21 \mathrm{E}-07)$ & $6.28 \mathrm{E}-05$ & $(8.07 \mathrm{E}-07)$ & & & $5.58 \mathrm{E}-05$ & $(2.00 \mathrm{E}-09)$ \\
\hline $\mathrm{E}\left[h_{y, t}\right]$ & & & & & $1.63 \mathrm{E}+00$ & $(4.91 \mathrm{E}-02)$ & $1.80 \mathrm{E}+00$ & $(1.06 \mathrm{E}+00)$ \\
\hline$\chi$ & & & $-6.06 \mathrm{E}-03$ & $(1.51 \mathrm{E}-04)$ & & & $-5.67 \mathrm{E}-03$ & $(1.69 \mathrm{E}-04)$ \\
\hline$\nu_{2}$ & & & & & $1.33 \mathrm{E}-05$ & $(2.54 \mathrm{E}+02)$ & & \\
\hline$\nu_{3}$ & & & & & & & $9.81 \mathrm{E}-05$ & $(2.74 \mathrm{E}+00)$ \\
\hline Model Properties & & & & & & & & \\
\hline Average Physical Volatility & 18.34 & & 18.34 & & 18.34 & & 18.34 & \\
\hline Average Model IV & 20.77 & & 20.79 & & 21.09 & & 21.00 & \\
\hline Volatility Persistence & & & & & & & & \\
\hline From Returns & 0.9911 & & & & & & & \\
\hline From RV & & & 0.9878 & & 0.9864 & & & \\
\hline From RBV & & & & & & & 0.9890 & \\
\hline From RJV & & & & & & & 0.9958 & \\
\hline Log-Likelihoods & & & & & & & & \\
\hline Returns, RBV, RJV, and Options & & & & & & & 156,970 & \\
\hline Returns & 19,019 & & 19,191 & & 18,955 & & 18,695 & \\
\hline Returns and Options & 52,770 & & 56,214 & & 55,550 & & 56,529 & \\
\hline Option Errors & & & & & & & & \\
\hline IVRMSE & 5.74 & & 5.04 & & 5.05 & & 4.77 & \\
\hline Ratio to GARCH & 1.000 & & 0.878 & & 0.879 & & 0.831 & \\
\hline VWRMSE & 4.96 & & 4.25 & & 4.37 & & 4.09 & \\
\hline Ratio to GARCH & 1.000 & & 0.858 & & 0.881 & & 0.825 & \\
\hline
\end{tabular}

\section{Model Properties}

Notes: Using daily returns, daily realized variation measures and options, we estimate our four models using a joint maximum likelihood criterion. The table reports the joint likelihood value as well as its decomposition into the various components. Option errors are reported using implied volatility root mean squared errors (IVRMSE) and vega-weighted root mean squared errors (VWRMSE) as defined in the text. The sample is from 2 January 1996 through 28 August 2013. Standard errors are reported in parentheses. Physical variance targeting is used to fix the $\omega$ parameters. 
Table 4: Implied Volatility Root Mean Squared Error (IVRMSE) by Moneyness, Maturity and VIX Level. 1996-2013

\begin{tabular}{ccccccc}
\multicolumn{7}{c}{ Panel A: IVRMSE by Moneyness } \\
Model & Delta $<0.3$ & $\frac{0.3<\text { Delta }<0.4}{0.4<\text { Delta }<0.5}$ & $\frac{0.5<\text { Delta }<0.6}{3.823}$ & $\frac{0.6<\text { Delta }<0.7}{3.896}$ & $\frac{1}{\text { Delta }>0.7}$ \\
GARCH & 5.338 & 4.001 & 2.970 & 3.139 & 3.228 & 8.112 \\
RVM & 4.671 & 3.226 & 3.214 & 3.070 & 3.389 & 7.364 \\
JVM & 5.059 & 3.568 & 2.825 & 2.956 & 3.319 & 6.821
\end{tabular}

\begin{tabular}{ccccccc} 
Model & \multicolumn{7}{c}{ Panel B: IVRMSE by Maturity } \\
GARCH & 5.259 & $\frac{30<\mathrm{DTM}<60}{5.700}$ & $\frac{60<\mathrm{DTM}<90}{5.640}$ & $\frac{90<\mathrm{DTM}<120}{5.852}$ & $\frac{120<\mathrm{DTM}<150}{6}$ & $\frac{\mathrm{DTM}>150}{5.497}$ \\
RVM & 4.531 & 4.985 & 5.003 & 4.894 & 5.856 & 5.300 \\
JVM & 4.437 & 4.731 & 4.956 & 5.078 & 6.115 & 5.755 \\
BPJVM & 4.404 & 4.731 & 4.739 & 4.555 & 5.535 & 4.948
\end{tabular}

\begin{tabular}{ccccccc}
\multicolumn{7}{c}{ Panel C: IVRMSE by VIX Level } \\
Model & $\frac{\mathrm{VIX}<15}{4.310}$ & $\frac{15<\mathrm{VIX}<20}{3.419}$ & $\frac{20<\mathrm{VIX}<25}{5.499}$ & $\frac{25<\mathrm{VIX}<30}{6.785}$ & $\frac{30<\mathrm{VIX}<35}{6.961}$ & $\frac{\text { VIX }>35}{11.639}$ \\
GARCH & 3.565 & 3.201 & 5.455 & 6.434 & 6.443 & 7.497 \\
RVM & 3.565 & 3.220 & 5.466 & 6.356 & 6.357 & 7.463 \\
JVM & 3.956 & 3.202 & 5.358 & 5.868 & 5.888 & 7.231
\end{tabular}

Notes: We use the parameter values in Table 3 to fit our four models to the 21,283 S\&P500 index option contracts from OptionMetrics. The top panel reports IVRMSE for contracts sorted by moneyness defined using the Black-Scholes delta. The second panel reports IVRMSE for contracts sorted by days to maturity (DTM). The third panel reports the IVRMSE for contracts sorted by the VIX level on the day corresponding to the option quote. 